\title{
A calculation of the multiplicative character
}

\author{
Jens Kaad
}

\begin{abstract}
We give an explicit formula for the application of the Connes-Karoubi multiplicative character to higher Loday products of elements in the connected component of the identity. On our way we construct a product on the relative $K$-groups of Banach algebras and investigate the multiplicative properties of the relative Chern character.
\end{abstract}

Mathematics Subject Classification (2010). 19D55, 19D45, 46L80.

Keywords. Algebraic K-theory, secondary invariants, Chern characters.

\section{Introduction}

To each $n$-summable Fredholm module $(F, H)$ over a $\mathbb{C}$-algebra $A$, A. Connes and M. Karoubi associate a multiplicative character on algebraic K-theory:

$$
\mathcal{M}_{F}: K_{n}(A) \rightarrow \mathbb{C} /(2 \pi i)^{\left\lceil\frac{n}{2}\right\rceil} \mathbb{Z} .
$$

The construction uses the relative $K$-groups of a Banach algebra and the relative Chern character with values in continuous cyclic homology. It can be understood as a pairing between the abelian group generated by finitely summable Fredholm modules and algebraic K-theory, [9].

In the case where $n=1$ the multiplicative character has a direct interpretation as a Fredholm determinant, see [9]; likewise, in the case where $n=2$ the multiplicative character coincides with the determinant invariant as defined in [2], [3], see [15]. This implies the independence of the multiplicative character under trace class perturbations. Furthermore, when $A$ is a commutative Banach algebra we have the explicit formula

$$
\mathcal{M}_{F}\left(\left[e^{a}\right] *\left[e^{b}\right]\right)=-q(\operatorname{Tr}[P a P, P b P]) \in \mathbb{C} /(2 \pi i) \mathbb{Z}, \quad a, b \in A,
$$

for the application of the multiplicative character to the Loday product $\left[e^{a}\right] *\left[e^{b}\right] \in$ $K_{2}(A)$. The operator $P=(F+1) / 2 \in \mathscr{L}(H)$ is the projection onto the eigenvectors with eigenvalue 1 of the selfadjoint unitary $F \in \mathscr{L}(H)$. The map $q: \mathbb{C} \rightarrow \mathbb{C} /(2 \pi i) \mathbb{Z}$ is the quotient map and $\operatorname{Tr}: \mathscr{L}^{1}(P H) \rightarrow \mathbb{C}$ is the operator trace on the trace ideal. With these low-dimensional interpretations in mind we could try to think of the 
multiplicative character as a higher determinant on algebraic K-theory. The aim of the present paper is then to find an analogue of the formula (1.1) in higher dimensions. This pursuit could be justified by the amount of research which focus on the quantity $\operatorname{Tr}[P a P, P b P] \in \mathbb{C}$, see for example [4], [5], [13]. We would also like to mention the use of the determinant invariant in relation with generalizations of the Szegö limit theorem, [6].

Let us fix a continuous $n$-summable Fredholm module $(F, H)$ over a unital commutative Banach algebra $A$. The interior Loday product makes the direct sum of algebraic $K$-groups $\bigoplus_{k=1}^{\infty} K_{k}(A)$ into a graded commutative ring; see [20]. Let us choose some elements $a_{0}, \ldots, a_{n-1} \in M_{\infty}(A)$ and form their exponentials $e^{a_{0}}, \ldots, e^{a_{n-1}} \in \mathrm{GL}_{0}(A)$. Each of them determines a class in the first algebraic $K$-group and we can consider their Loday product

$$
[x]=\left[e^{a_{0}}\right] * \cdots *\left[e^{a_{n-1}}\right] \in K_{n}(A) .
$$

The main result of the present paper is then the explicit formula

$$
\mathcal{M}_{F}([x])=(-1)^{n} \sum_{s \in \Sigma_{n-1}} \operatorname{sgn}(s)\left(q \circ \tau_{F}\right)\left(\operatorname{TR}\left(a_{0}\right) \otimes \operatorname{TR}\left(a_{s(1)}\right) \otimes \cdots \otimes \operatorname{TR}\left(a_{s(n-1)}\right)\right)
$$

for the application of the multiplicative character to this concrete element in higher K-theory. The linear map TR: $M_{\infty}(A) \rightarrow A$ is the matrix trace and $q: \mathbb{C} \rightarrow$ $\mathbb{C} /(2 \pi i)^{\lceil n / 2\rceil} \mathbb{Z}$ is the quotient map. The homomorphism

$$
\begin{aligned}
\tau_{F}: C_{n-1}^{\text {cont }}(A) / \operatorname{Im}(1-t) & \rightarrow \mathbb{C}, \\
\left(a_{0}, \ldots, a_{n-1}\right) & \mapsto c_{n-1} \operatorname{Tr}\left(\gamma^{n} F\left[F, a_{0}\right] \ldots\left[F, a_{n-1}\right]\right),
\end{aligned}
$$

is the continuous index cocycle associated with the continuous $n$-summable Fredholm module; see [8], Section IV.1. Here $c_{n-1} \in \mathbb{Q}$ is some rational constant and $\gamma \in$ $\mathscr{L}(H)$ is the grading operator of the Fredholm module. In case $n=2 m$ is even, the above formula can be expressed in terms of the higher fundamental trace form of J. W. Helton and R. E. Howe. Indeed,

$$
\begin{gathered}
\sum_{s \in \Sigma_{2 m-1}} \operatorname{sgn}(s) \tau_{F}\left(\operatorname{TR}\left(a_{0}\right) \otimes \operatorname{TR}\left(a_{s(1)}\right) \otimes \cdots \otimes \operatorname{TR}\left(a_{s(2 m-1)}\right)\right) \\
=-\frac{1}{m !}\left\langle P \operatorname{TR}\left(a_{0}\right) P, \ldots, P \operatorname{TR}\left(a_{2 m-1}\right) P\right\rangle,
\end{gathered}
$$

where we recall that the trace form is given by

$$
\langle\cdot, \ldots, \cdot\rangle:\left(x_{1}, \ldots, x_{2 m}\right) \mapsto \operatorname{Tr}\left(\sum_{s \in \Sigma_{2 m}} \operatorname{sgn}(s) x_{s(1)} \ldots x_{s(2 m)}\right)
$$

on suitable algebras; see [13], part II, §2, and [7], part I, §7. This shows that the multiplicative character is computable on the subgroup of $K_{n}(A)$ generated by Loday products of elements in the connected component of the identity. Note that the commutativity assumption serves to ensure the existence of the interior Loday product which is needed for the calculation to make sense. 
Let us reflect a bit on what we have obtained. First of all, we could choose different "logarithms" for the exponentials $e^{a_{0}}, \ldots, e^{a_{n-1}} \in \mathrm{GL}_{0}(A)$. Thus, let $b_{0}, \ldots, b_{n-1} \in M_{\infty}(A)$ be any elements such that $e^{b_{i}}=e^{a_{i}}$ for all $i \in\{0, \ldots, n-1\}$. We then get that the difference

$$
\begin{aligned}
& \sum_{s \in \Sigma_{n-1}} \operatorname{sgn}(s)\left(\tau_{F}\left(a_{0} \otimes a_{s(1)} \otimes \cdots \otimes a_{s(n-1)}\right)\right. \\
& \left.\quad-\tau_{F}\left(b_{0} \otimes b_{s(1)} \otimes \cdots \otimes b_{s(n-1)}\right)\right) \in(2 \pi i)^{\lceil n / 2\rceil} \mathbb{Z}
\end{aligned}
$$

lies in the additive subgroup $(2 \pi i)^{\lceil n / 2\rceil} \mathbb{Z} \subseteq \mathbb{C}$. This is an immediate corollary of the formula (1.2). Furthermore, the properties of the Loday product yields a couple of desirable properties for the map

$$
d_{F}: \operatorname{GL}(A)^{n} \rightarrow \mathbb{C} /(2 \pi i)^{\lceil n / 2\rceil} \mathbb{Z}, \quad\left(g_{0}, \ldots, g_{n-1}\right) \mapsto \mathcal{M}_{F}\left(\left[g_{0}\right] * \cdots *\left[g_{n-1}\right]\right) .
$$

To be more precise:

(1) It is multilinear:

$$
d_{F}\left(g_{0}, \ldots, g_{i} \cdot h_{i}, \ldots, g_{n-1}\right)=d_{F}\left(g_{0}, \ldots, g_{n-1}\right)+d_{F}\left(g_{0}, \ldots, h_{i}, \ldots, g_{n-1}\right) .
$$

(2) It is antisymmetric:

$$
d_{F}\left(g_{s(0)}, \ldots, g_{s(n-1)}\right)=\operatorname{sgn}(s) d_{F}\left(g_{0}, \ldots, g_{n-1}\right), \quad s \in \Sigma_{n} .
$$

(3) It vanishes whenever one of the entries is an elementary matrix:

$$
d_{F}\left(g_{0}, \ldots, e_{i}, \ldots, g_{n-1}\right)=0, \quad e_{i} \in E(A) .
$$

See [20]. We will think of the $\operatorname{map} d_{F}: \operatorname{GL}(A)^{n} \rightarrow \mathbb{C} /(2 \pi i)^{\lceil n / 2\rceil} \mathbb{Z}$ as a higher determinant associated with the continuous $n$-summable Fredholm module $(F, H)$ over the unital commutative Banach algebra $A$. This is justified by the low-dimensional case, the properties listed above, and the concrete expression in terms of logarithms and the operator trace on Loday products of elements in the connected component of the identity; see (1.2).

The similiarity of the trace formula in (1.2) with the expression in the bivariant case, makes us expect the following generalizations: First of all the quantity $\left\langle P \operatorname{TR}\left(a_{0}\right) P, \ldots, P \operatorname{TR}\left(a_{2 m-1}\right) P\right\rangle \in \mathbb{C}$ should be invariant under perturbations of the operators $P \operatorname{TR}\left(a_{i}\right) P$ by elements in the Schatten ideal $\mathscr{L}^{m}(P H)$. Furthermore, under suitable conditions, the form should be expressible by means of an integral over the joint essential spectrum of the operators in question. In this respect, see [13], part II, and [8], Section IV.2. $\gamma$, Theorem 8, p. 308.

At this point, we would like to explain briefly how the main result is obtained. Let us assume that $A$ is a unital commutative Banach algebra. In order to calculate the multiplicative character of some element $[x] \in K_{n}(A)$ the first obstacle is to construct a lift in relative $\mathrm{K}$-theory,

$$
[\gamma] \in K_{n}^{\mathrm{rel}}(A), \quad \theta[\gamma]=[x] .
$$


Let us assume that our element $[x] \in K_{n}(A)$ is defined as the Loday product of elements represented by invertibles in the connected component of the identity

$$
[x]=\left[g_{0}\right] * \cdots *\left[g_{n-1}\right] \in K_{n}(A), \quad g_{0}, \ldots, g_{n-1} \in \mathrm{GL}_{0}(A) .
$$

The contractibility assumption on our representatives entails that there exists a lift of each of them in the first relative $K$-group. Let us choose some lifts

$$
\left[\gamma_{0}\right], \ldots,\left[\gamma_{n-1}\right] \in K_{1}^{\mathrm{rel}}(A), \quad \theta\left[\gamma_{i}\right]=\left[g_{i}\right] .
$$

To find a lift of the element $[x] \in K_{n}(A)$ we construct an explicit product on the relative $K$-groups. This product makes the direct sum of relative $K$-groups $\bigoplus_{k=1}^{\infty} K_{k}^{\text {rel }}(A)$ into a graded commutative ring and the map

$$
\theta: \bigoplus_{k=1}^{\infty} K_{k}^{\mathrm{rel}}(A) \rightarrow \bigoplus_{k=1}^{\infty} K_{k}(A)
$$

becomes a homomorphism of graded rings (recall that $A$ is assumed to be commutative), see Theorem 3.18. The desired lift $[\gamma] \in K_{n}^{\text {rel }}(A)$ is then given by the relative Loday product of the individual lifts

$$
[\gamma]=\left[\gamma_{0}\right] *^{\mathrm{rel}} \cdots *^{\mathrm{rel}}\left[\gamma_{n-1}\right], \quad \theta[\gamma]=[x] .
$$

On our way we also express the second relative $K$-group as the second homology group of a certain simplicial set, see Corollary 3.8.

Having found the lift $[\gamma] \in K_{n}^{\text {rel }}(A)$ the next problem is to calculate the relative Chern character of this lift

$$
\mathrm{ch}^{\mathrm{rel}}: K_{n}^{\mathrm{rel}}(A) \rightarrow \mathrm{HC}_{n-1}(A), \quad \mathrm{ch}^{\mathrm{rel}}[\gamma]=? .
$$

Following the same vein of ideas we show in Theorem 4.9 that the relative Chern character is a homomorphism of graded rings. This should be understood in the following sense: The relative Chern character has degree minus one, so the corresponding product in continuous cyclic homology has degree plus one,

$$
*: \mathrm{HC}_{k-1}(A) \otimes \mathbb{C} \mathrm{HC}_{m-1}(A) \rightarrow \mathrm{HC}_{k+m-1}(A), \quad x * y=x \times(s N)(y) .
$$

The operator $N: C_{m-1}(A) \rightarrow C_{m-1}(A)$ is the norm operator and the operator $s: C_{m-1}(A) \rightarrow C_{m}(A)$ is the extra degeneracy. The map $\times: C_{k-1}(A) \times C_{m}(A) \rightarrow$ $C_{m+k-1}(A)$ is the interior Hochschild shuffle product; see also [21]. The calculation in question thus reduces to the case of $\mathrm{ch}^{\mathrm{rel}}: K_{1}^{\mathrm{rel}}(A) \rightarrow \mathrm{HC}_{0}(A)$. The elements in $K_{1}^{\mathrm{rel}}(A)$ are represented by smooth maps $\sigma:[0,1] \rightarrow \operatorname{GL}(A)$ which send 0 to the identity $1 \in \mathrm{GL}(A)$. The relative Chern character essentially determines the corresponding logarithm of the endpoint $\sigma(1) \in \mathrm{GL}_{0}(A)$. The desired formula (1.2) is now proved without too much effort, see Theorem 5.2. 
The paper is organized as follows: In Section 2 we give an account of the various product structures which will be used throughout the paper. We continue in Section 3 by constructing the product in relative K-theory and we give a proof of its main properties. In Section 4 we study the multiplicative properties of the relative Chern character with values in continuous cyclic homology. Finally, in Section 5, we show how our results lead to a calculation of the Connes-Karoubi multiplicative character on higher Loday products.

Acknowledgements. I would like to thank Ryszard Nest for his continuous support and many helpful comments. Furthermore, I would like to thank Jerome Kaminker for the nice talk we had at the U.C. Davis on the subject of the paper. I am also grateful to Max Karoubi for giving me some valuable indications related to his geometric viewpoint. Finally, I would like to thank the referee for some good suggestions that allowed me to improve the paper on some important points.

\section{A preliminary on various product structures in homology}

2.1. The exterior shuffle product. Let $A$ and $B$ be unital Banach algebras. We let $A \hat{\otimes} B$ denote the projective tensor product of $A$ and $B$ in the sense of Grothendieck, [12]. The definition of the simplicial sets $R_{p}(A)$ can be found in Section 3.

For each $p, q \in \mathbb{N}$ we fix an isomorphism $\varphi: A^{p} \otimes_{\mathbb{Z}} B^{q} \rightarrow\left(A \otimes_{\mathbb{Z}} B\right)^{p q}$ of $\left(A \otimes_{\mathbb{Z}} B\right)$-bimodules. We then have the associated group homomorphisms

$$
\otimes_{\varphi}: \mathrm{GL}_{p}(A) \times \mathrm{GL}_{q}(B) \rightarrow \mathrm{GL}_{p q}\left(A \otimes_{\mathbb{Z}} B\right)
$$

and

$$
\hat{\otimes}_{\varphi}=\iota \circ \otimes_{\varphi}: \mathrm{GL}_{p}(A) \times \mathrm{GL}_{q}(B) \rightarrow \mathrm{GL}_{p q}(A \hat{\otimes} B) .
$$

Here $\iota: \mathrm{GL}_{p q}\left(A \otimes_{\mathbb{Z}} B\right) \rightarrow \mathrm{GL}_{p q}(A \hat{\otimes} B)$ is induced by the "canonical" homomorphism $\iota: A \otimes_{\mathbb{Z}} B \rightarrow A \hat{\otimes} B$.

A pointwise version of the completed tensor product yields a map of simplicial sets

$$
\hat{\otimes}_{\varphi}: R_{p}(A) \times R_{q}(B) \rightarrow R_{p q}(A \hat{\otimes} B), \quad(\sigma, \tau) \mapsto\left(t \mapsto \sigma(t) \hat{\otimes}_{\varphi} \tau(t)\right) .
$$

Composition with the shuffle map [23]

$$
\text { sh: } C_{*}\left(R_{p}(A)\right) \otimes C_{*}\left(R_{q}(B)\right) \rightarrow C_{*}\left(R_{p}(A) \times R_{q}(B)\right)
$$

therefore equips us with a chain map

$$
\times_{\varphi}=\hat{\otimes}_{\varphi} \circ \mathrm{sh}: C_{*}\left(R_{p}(A)\right) \otimes C_{*}\left(R_{q}(B)\right) \rightarrow C_{*}\left(R_{p q}(A \hat{\otimes} B)\right) .
$$

The notation $C_{*}(X)$ refers to the chain complex with integer coefficients associated to any simplicial set $X$. The formal sum of smooth maps

$$
\sigma \times_{\varphi} \tau=\sum_{(\mu, \nu) \in \Sigma_{n, m}} \operatorname{sgn}(\mu, \nu) s_{\nu(m-1)} \ldots s_{\nu(0)}(\sigma) \hat{\otimes}_{\varphi} s_{\mu(n-1)} \ldots s_{\mu(0)}(\tau)
$$


will be called the exterior shuffle product of $\sigma \in R_{p}(A)_{n}$ and $\tau \in R_{q}(B)_{m}$. Here $\Sigma_{(n, m)} \subseteq \Sigma_{n+m}$ denotes the set of $(n, m)$-shuffles. We will show in Lemma 3.9 that the induced map on homology

$$
\times: \mathrm{H}_{n}\left(R_{p}(A)\right) \otimes \mathrm{H}_{m}\left(R_{q}(B)\right) \rightarrow \mathrm{H}_{n+m}\left(R_{p q}(A \hat{\otimes} B)\right)
$$

is independent of the choice of isomorphism $\varphi: A^{p} \otimes_{\mathbb{Z}} B^{q} \rightarrow\left(A \otimes_{\mathbb{Z}} B\right)^{p q}$.

2.2. The exterior wedge product in Lie algebra homology. Let $A$ and $B$ be unital Banach algebras. For each $n \geq 2$ we let $\wedge_{n} A$ denote the Banach space

$$
\bigwedge_{n} A:=\underbrace{A \hat{\otimes} \ldots \hat{\otimes} A}_{n} / \operatorname{ker}(S) \text {. }
$$

Here the closed subspace $\operatorname{ker}(S) \subseteq A^{\hat{\otimes} n}$ is the kernel of the continuous map

$$
S: A^{\widehat{\otimes} n} \rightarrow A^{\hat{\otimes} n}, \quad S\left(a_{1} \otimes \cdots \otimes a_{n}\right)=\sum_{\sigma \in \Sigma_{n}} \operatorname{sgn}(\sigma) a_{\sigma^{-1}(1)} \otimes \cdots \otimes a_{\sigma^{-1}(n)} .
$$

For $n=1$ we let $\wedge_{1} A=A$. Observe that the Banach space $\wedge_{n} A$ identifies with the quotient of $A^{\hat{\otimes} n}$ by the usual signed action of the symmetric group. The reason for using the above description is, that it becomes clear that $\wedge_{n} A$ is actually a Banach space for each $n \in \mathbb{N}$. This will turn out to be important for us in the sequel.

By the continuous Lie algebra homology of the unital Banach algebra $A$ we will then understand the homology of the chain complex $\left(\Lambda_{*} A, \delta\right)$. Here $\delta: \Lambda_{n} A \rightarrow$ $\Lambda_{n-1} A$ is the Chevalley-Eilenberg boundary map of $A$ regarded as a Banach Lie algebra.

We let

$$
\cdot \otimes 1_{B}: \bigwedge_{*} A \rightarrow \bigwedge_{*}(A \hat{\otimes} B) \quad \text { and } \quad 1_{A} \otimes \cdot: \bigwedge_{*} B \rightarrow \bigwedge_{*}(A \hat{\otimes} B)
$$

denote the chain maps obtained by functoriality from the continuous algebra homomorphisms

$$
a \mapsto a \otimes 1_{B} \quad \text { and } \quad b \mapsto 1_{A} \otimes b .
$$

We then have a chain map

$$
\wedge^{E}: \bigwedge_{*} A \otimes \bigwedge_{*} B \rightarrow \bigwedge_{*}(A \hat{\otimes} B)
$$

defined by

$$
x \otimes y \mapsto\left(x \otimes 1_{B}\right) \wedge\left(1_{A} \otimes y\right), \quad x \in \bigwedge_{n} A, y \in \bigwedge_{m} B .
$$

For each $x \in \wedge_{n} A$ and each $y \in \bigwedge_{m} B$ we will refer to the element

$$
x \wedge^{E} y:=\left(x \otimes 1_{B}\right) \wedge\left(1_{A} \otimes y\right) \in \bigwedge_{n+m} A \hat{\otimes} B
$$


as the exterior wedge product of $x$ and $y$. We let

$$
\wedge^{E}: \mathrm{H}_{n}^{\mathrm{Lie}}(A) \otimes \mathrm{H}_{m}^{\mathrm{Lie}}(B) \rightarrow \mathrm{H}_{n+m}^{\mathrm{Lie}}(A \hat{\otimes} B)
$$

denote the induced map on continuous Lie algebra homology. The exterior wedge product, thus defined, is seen to be associative and graded commutative at the level of complexes.

2.3. The exterior product of degree one in cyclic homology. Let $A$ and $B$ be unital Banach algebras. We let

$$
\times: C_{*}(A) \otimes C_{*}(B) \rightarrow C_{*}(A \hat{\otimes} B)
$$

denote the exterior shuffle product on the continuous Hochschild complex, [21], Section 4.2. Furthermore, we let $\left(C_{*}^{\lambda}(A), b\right)$ denote the continuous cyclic complex. Thus in each degree $n \in \mathbb{N} \cup\{0\}$ we have a Banach space $C_{n}^{\lambda}(A)$, [9], [17]. Observe that the image $\operatorname{Im}(1-t) \subseteq A \hat{\otimes} A^{\hat{\otimes} n}$ is closed since it coincides with the kernel of the norm operator $N: A \hat{\otimes} A^{\hat{\otimes} n} \rightarrow A \hat{\otimes} A^{\hat{\otimes} n}$.

By the exterior product of degree one in continuous cyclic homology we will understand the map

$$
*: C_{n}^{\lambda}(A) \otimes C_{m}^{\lambda}(B) \rightarrow C_{n+m+1}^{\lambda}(A \hat{\otimes} B)
$$

defined by

$$
x * y=x \times(s N y), \quad x \in C_{n}^{\lambda}(A), y \in C_{m}^{\lambda}(B) .
$$

Here $N: C_{m}(B) \rightarrow C_{m}(B)$ is the norm operator $N=1+t+\cdots+t^{m}$ and $s: C_{m}(B) \rightarrow C_{m+1}(B)$ is the extra degeneracy.

We will need to show that the product is well defined. For this, consider the map

$$
E: C_{n}(A) \rightarrow \bigwedge_{n+1} M_{n+1}(A), \quad\left(a_{0}, \ldots, a_{n}\right) \mapsto E_{12}\left(a_{0}\right) \wedge \cdots \wedge E_{(n+1) 1}\left(a_{n}\right),
$$

where $E_{i j}(a)$ denotes the matrix with $a \in A$ in position $(i, j)$ and zeros elsewhere, [22], [30].

From Theorem 4.4 and Theorem 4.5 we then get the equality

$$
x * y=(\mathrm{TR} \circ \varepsilon)\left(E(x) \wedge^{E} E(y)\right), \quad x \in C_{n}(A), y \in C_{m}(B),
$$

where $\varepsilon: \bigwedge_{*} M_{k}(A) \rightarrow C_{*-1}^{\lambda}\left(M_{k}(A)\right)$ and TR: $C_{*}^{\lambda}\left(M_{k}(A)\right) \rightarrow C_{*}^{\lambda}(A)$ denote the antisymmetrization map and the generalized trace on continuous cyclic homology respectively. It follows that the product is well defined and that it is associative and graded commutative at the level of complexes.

Lastly, the Hochschild boundary is a (shifted) graded derivation with respect to the product

$$
b(x * y)=(b x) * y+(-1)^{\operatorname{deg}(x)+1} x *(b y) .
$$


It follows that our multiplication descends to an exterior product of degree one on continuous cyclic homology

$$
*: \mathrm{HC}_{n}(A) \otimes \mathbb{C} \mathrm{HC}_{m}(B) \rightarrow \mathrm{HC}_{n+m+1}(A \hat{\otimes} B) .
$$

It should be remarked that if the operator $s N: C_{m}(B) \rightarrow C_{m+1}(B)$ is replaced by the Connes boundary $B=(1-t) s N: C_{m}(B) \rightarrow C_{m+1}(B)$ in (2.1), we get the same exterior product of degree one on cyclic homology. However, using the Connes boundary, the product does not become associative at the level of complexes.

In the case where the unital Banach algebra $A$ is commutative we get an interior product

$$
\text { *: } \mathrm{HC}_{n}(A) \otimes_{\mathbb{C}} \mathrm{HC}_{m}(A) \rightarrow \mathrm{HC}_{n+m+1}(A)
$$

by composition of the exterior product with the map induced by the multiplication $\nabla: A \hat{\otimes} A \rightarrow A$.

For further details on the constructions given in this section we refer to [14], [21], [31].

\section{An exterior product on the relative K-theory of Banach algebras}

Let $A$ be a unital Banach algebra. Before giving the construction of the exterior product, we recall the definition of the relative $K$-groups, as introduced by M. Karoubi, [17].

For each $n \in \mathbb{N}_{0}$ we let $\Delta^{n}$ denote the presentation

$$
\Delta^{n}= \begin{cases}\left\{\left(t_{1}, \ldots, t_{n}\right) \in \mathbb{R}^{n} \mid t_{i} \geq 0, \sum_{i=1}^{n} t_{i} \leq 1\right\}, & n \geq 1, \\ \{0\} \in \mathbb{R}, & n=0,\end{cases}
$$

of the standard $n$-simplex. The vertices $v_{0}, \ldots v_{n} \in \Delta^{n}$ are given by

$$
v_{i}= \begin{cases}(0, \ldots, 1, \ldots, 0), & i \in\{1, \ldots, n\}, \\ (0, \ldots, 0), & i=0 .\end{cases}
$$

Notice that we do not use the standard presentation of the standard $n$-simplex. The reason for our alternative choice is, that we want to emphasize the special role of the zeroth vertex $v_{0} \in \Delta^{n}$, see the formulas below.

To each $p \in \mathbb{N} \cup\{\infty\}$ we associate a simplicial set $R_{p}(A)$. In degree $n \in \mathbb{N}_{0}$ it is given by the set of normalized smooth maps

$$
\sigma: \Delta^{n} \rightarrow \mathrm{GL}_{p}(A), \quad \sigma\left(v_{0}\right)=1_{p} .
$$

Here the word "smooth" means that $\sigma=\left.\tilde{\sigma}\right|_{\Delta^{n}}$ is obtained as the restriction of some smooth map with compact support $\tilde{\sigma} \in C_{c}^{\infty}\left(\mathbb{R}^{n}, M_{p}(A)\right)$. 
The face operators and degeneracy operators are given by

$$
\begin{aligned}
& d_{i}(\sigma)\left(t_{1}, \ldots, t_{n-1}\right)= \begin{cases}\sigma\left(1-\sum_{j=1}^{n-1} t_{j}, t_{1}, \ldots, t_{n-1}\right) \cdot \sigma\left(v_{1}\right)^{-1} & \text { for } i=0, \\
\sigma\left(t_{1}, \ldots, t_{i-1}, 0, t_{i}, \ldots, t_{n-1}\right) & \text { for } i \in\{1, \ldots, n\},\end{cases} \\
& s_{j}(\sigma)\left(t_{1}, \ldots, t_{n+1}\right)= \begin{cases}\sigma\left(t_{2}, \ldots, t_{n+1}\right) & \text { for } j=0, \\
\sigma\left(t_{1}, \ldots, t_{j-1}, t_{j}+t_{j+1}, \ldots, t_{n+1}\right) & \text { for } j \in\{1, \ldots, n\} .\end{cases}
\end{aligned}
$$

Notice the extra factor $\sigma\left(v_{1}\right)^{-1}$ in the expression for $d_{0}: R_{p}(A)_{n} \rightarrow R_{p}(A)_{n-1}$. We will often refer to the simplicial set $R_{\infty}(A)$ by $R(A)$.

Remark 3.1. The simplicial set $R_{p}(A)$ is isomorphic to the simplicial set $\mathrm{GL}_{p}\left(A_{*}\right) / \mathrm{GL}_{p}(A)$ which is applied by M. Karoubi in his definition of relative Ktheory. See [17], §6.18. The isomorphism is given by the quotient map.

It can be proved that the simplicial set $R_{p}(A)$ is a pointed Kan complex with fundamental group

$$
\pi_{1}\left(R_{p}(A)\right)=R_{p}(A)_{1} / \sim .
$$

Here $\sim$ denotes the equivalence relation of smooth homotopies with fixed end points. The group structure is given by pointwise multiplication. Furthermore, for each $p \in\{3,4, \ldots\} \cup\{\infty\}$ let

$$
\left(F_{p}(A)_{1} / \sim\right) \subseteq \pi_{1}\left(R_{p}(A)\right) .
$$

denote the normal closure of the subgroup generated by the elementary matrices

$$
e_{i j}(\rho) \in R_{p}(A), \quad \rho \in C^{\infty}\left([0,1], M_{p}(A)\right), \rho(0)=0 .
$$

The group $F_{p}(A)_{1} / \sim$ is seen to be perfect and when $p=\infty$ it coincides with the commutator subgroup of $\pi_{1}\left(R_{\infty}(A)\right)$; see [17], §6.18. We can thus apply the plus construction to the geometric realization of $R_{p}(A)$ for all $p \in\{3,4, \ldots\} \cup\{\infty\}$.

Definition 3.2 ([17]). By the relative $K$-groups of the unital Banach algebra $A$ we will understand the homotopy groups of the pointed topological space $|R(A)|^{+}$,

$$
K_{n}^{\mathrm{rel}}(A):=\pi_{n}\left(|R(A)|^{+}\right), \quad n \geq 1 .
$$

It is then proved in [17], $\S 6.15-6.18$, that the relative $K$-groups relate the algebraic $K$-groups and topological $K$-groups through the long exact sequence

$$
\begin{aligned}
& \cdots \stackrel{i}{\longrightarrow} K_{n+1}^{\mathrm{top}}(A) \stackrel{v}{\longrightarrow} K_{n}^{\mathrm{rel}}(A) \stackrel{\theta}{\longrightarrow} K_{n}(A) \\
& \cdots \stackrel{i}{\stackrel{i}{\longleftarrow}} K_{n-1}(A) \stackrel{\theta}{\stackrel{\theta}{\longleftarrow}} K_{n-1}^{\mathrm{rel}}(A) \stackrel{v}{\longleftarrow} K_{n}^{\mathrm{top}}(A) .
\end{aligned}
$$


Here the map $\theta: K_{n}^{\mathrm{rel}}(A) \rightarrow K_{n}(A)$ is induced by the map of simplicial sets

$$
\theta: R_{p}(A) \rightarrow \mathrm{BGL}_{p}(A), \quad \sigma \mapsto\left(\sigma\left(v_{1}\right)^{-1}, \sigma\left(v_{1}\right) \sigma\left(v_{2}\right)^{-1}, \ldots, \sigma\left(v_{n-1}\right) \sigma\left(v_{n}\right)^{-1}\right) .
$$

3.1. A calculation of the second relative $\boldsymbol{K}$-group. In this section we show that the second relative $K$-group is isomorphic to the second homology group of a certain simplicial set. The result is thus similar to the result in [20], [25], [26]. Here the second algebraic $K$-group of a unital ring (using Quillen's definition) is shown to agree with the second homology group of the elementary matrices over the unital ring. The present calculation is relevant for our explicit definition of the exterior product on the relative $K$-groups.

Let $A$ be a unital Banach algebra. Let $F(A)_{1} \subseteq R(A)_{1}$ denote the smallest subgroup of $R(A)_{1}$ such that

$$
\sigma \tau \sigma^{-1} \tau^{-1} \in F(A)_{1}
$$

for all $\sigma, \tau \in R(A)_{1}$ which are constant on neighborhoods of the vertices $v_{0}, v_{1} \in$ $\Delta^{1} \subseteq \mathbb{R}$. Notice that $F(A)_{1}$ is perfect and normal in $R(A)_{1}$.

For $n \geq 2$ we then define the $n$-simplices of a simplicial set $F(A)$ by the recursive formula:

$$
\sigma \in F(A)_{n} \Longleftrightarrow \sigma \in R(A)_{n} \text { and }\left(d_{i}(\sigma) \in F(A)_{n-1} \text { for all } i \in\{0,1, \ldots, n\}\right) .
$$

The face and degeneracy operators are induced by the corresponding operators on the simplicial set $R(A)$. It can be proved that $F(A)$ is a Kan complex also.

Now let $\sim_{F}$ denote the equivalence relation on $R(A)$ which is defined degreewise by

$$
x \sim_{F} y \Longleftrightarrow \text { there exists } \tau \in F(A)_{n}: x \cdot \tau=y .
$$

Here the elements $x, y \in R(A)_{n}$ are assumed to be of degree $n \geq 1$. We define the simplicial set $Q(A):=R(A) / \sim_{F}$ as the quotient of $R(A)$ by the above equivalence relation.

Theorem 3.3. The quotient map $\pi: R(A) \rightarrow Q(A)$ is a Kan fibration.

Proof. Suppose that $x_{0}, \ldots, x_{k-1}, x_{k+1}, \ldots, x_{n} \in R(A)_{n-1}$ are compatible and suppose that there exist an $n$-simplex $y \in R(A)_{n}$ and $(n-1)$-simplices $\tau_{0}, \ldots, \tau_{k-1}$, $\tau_{k+1}, \ldots, \tau_{n} \in F(A)_{n-1}$ such that

$$
d_{j}(y) \tau_{j}=x_{j} \quad \text { for all } j \neq k .
$$

Using the compatibility of the simplices $x_{0}, \ldots, x_{k-1}, x_{k+1}, \ldots, x_{n} \in R(A)_{n-1}$ we obtain that

$$
d_{i}\left(\tau_{j}\right)= \begin{cases}d_{j-1}\left(\tau_{i}\right) & \text { for all } 0<i<j \leq n, i, j \neq k, \\ y\left(v_{1}\right)^{-1} d_{j-1}\left(\tau_{0}\right) y\left(v_{1}\right) & \text { for all } 0=i<j \leq n, i, j \neq k\end{cases}
$$


The $(n-1)$-simplices

$$
y\left(v_{1}\right)^{-1} \cdot \tau_{0} \cdot y\left(v_{1}\right), \tau_{1}, \ldots, \tau_{k-1}, \tau_{k+1}, \ldots, \tau_{n} \in F(A)_{n-1}
$$

are thus compatible. Since $F(A)$ is a Kan complex there exists an $n$-simplex $\tau \in$ $F(A)_{n}$ such that

$$
d_{j}(\tau)= \begin{cases}\tau_{j} & \text { for all } j \neq 0, k, \\ y\left(v_{1}\right)^{-1} \tau_{0} y\left(v_{1}\right) & \text { for } j=0, j \neq k .\end{cases}
$$

The $n$-simplex $z:=y \cdot \tau \in R(A)_{n}$ then satisfies the properties

$$
d_{j}(z)=x_{j} \text { for all } j \neq k \text { and } \pi(z)=\pi(y) .
$$

This proves that $\pi: R(A) \rightarrow Q(A):=R(A) / \sim_{F}$ is a Kan fibration.

The Kan complex $F(A)$ is the fiber of the Kan fibration $\pi: R(A) \rightarrow Q(A)$. The next result on the homotopy groups of the fiber can then be proved without too much effort.

Lemma 3.4. The inclusion $i: F(A) \rightarrow R(A)$ induces an isomorphism

$$
\pi_{n}(i): \pi_{n}(F(A)) \rightarrow \pi_{n}(R(A)) \text { for all } n \geq 2 .
$$

The fundamental group of the fiber $F(A)$ coincides with the commutator subgroup of $\pi_{1}(R(A))$ and the induced map

$$
\pi_{1}(i):\left[\pi_{1}(R(A)), \pi_{1}(R(A))\right] \rightarrow \pi_{1}(R(A))
$$

is the inclusion.

The existence of the long exact sequence of homotopy groups associated with the fibration

$$
F(A) \stackrel{i}{\rightarrow} R(A) \stackrel{\pi}{\rightarrow} Q(A)
$$

then allows us to calculate the homotopy groups of the quotient.

Corollary 3.5. The homotopy groups of $Q(A)$ are given by

$$
\pi_{n}(Q(A))= \begin{cases}0 & \text { for } n \geq 2, \\ \pi_{1}(R(A)) /\left[\pi_{1}(R(A)), \pi_{1}(R(A))\right] & \text { for } n=1 .\end{cases}
$$

The induced map $\pi_{1}(\pi): \pi_{1}(R(A)) \rightarrow \pi_{1}(Q(A))$ is the quotient map.

Let $\pi^{+}:|R(A)|^{+} \rightarrow|Q(A)|$ be a representative for the homotopy class of continuous maps obtained from the quotient map $\pi: R(A) \rightarrow Q(A)$ by universality of the plus-construction.

Let $\mathcal{F}$ denote the homotopy fiber of $\pi^{+}:|R(A)|^{+} \rightarrow|Q(A)|$. 
Theorem 3.6. The inclusion $i: F(A) \rightarrow R(A)$ gives rise to a homotopy equivalence

$$
f^{+}:|F(A)|^{+} \rightarrow \mathcal{F} \text {. }
$$

Proof. This follows from [1] since $\pi_{1}(Q(A))$ is abelian. Notice that the maximal perfect subgroups of both $\pi_{1}(F(A))$ and $\pi_{1}(R(A))$ are given by the commutator subgroups.

With this precise description of the homotopy fiber $\mathcal{F}$ in hand, we are able to obtain the desired calculation of the second relative $K$-group.

Corollary 3.7. The space $|F(A)|^{+}$is simply connected and the map

$$
i^{+}:|F(A)|^{+} \rightarrow|R(A)|^{+}
$$

induced by the inclusion $i: F(A) \rightarrow R(A)$ yields an isomorphism

$$
\pi_{n}\left(i^{+}\right): \pi_{n}\left(|F(A)|^{+}\right) \rightarrow \pi_{n}\left(|R(A)|^{+}\right)=K_{n}^{\mathrm{rel}}(A)
$$

for all $n \geq 2$.

Proof. This is immediate from the long exact sequence of homotopy groups arising from the fibration $|F(A)|^{+} \rightarrow|R(A)|^{+} \rightarrow|Q(A)|$.

Corollary 3.8. The second relative $K$-group of the unital Banach algebra $A$ is isomorphic to the second homology group of the simplicial set $F(A)$,

$$
K_{2}^{\mathrm{rel}}(A) \cong H_{2}(F(A)) \text {. }
$$

Proof. Since $|F(A)|^{+}$is simply connected the Hurewicz homomorphism

$$
h_{2}: \pi_{2}\left(|F(A)|^{+}\right) \rightarrow H_{2}\left(|F(A)|^{+}\right) \cong H_{2}(|F(A)|) \cong H_{2}(F(A))
$$

is an isomorphism. But the group $\pi_{2}\left(|F(A)|^{+}\right)$is isomorphic to $K_{2}^{\text {rel }}(A)$ by Corollary 3.7 .

3.2. An $\boldsymbol{H}$-group structure on $|\boldsymbol{R}(\boldsymbol{A})|^{+}$. In this section we show that a pointwise version of the direct sum on $\operatorname{GL}(A)$ determines a commutative $H$-group structure on $|R(A)|^{+}$. Our exposition will follow [20], Section 1.2, and [32] closely.

Let $A$ be a unital Banach algebra. We define the direct sum on the simplicial set $R(A)$ as a pointwise version of the direct sum on $\operatorname{GL}(A)$, thus

$$
\oplus: R(A) \times R(A) \rightarrow R(A), \quad(\sigma, \tau) \mapsto(t \mapsto \sigma(t) \oplus \tau(t)) .
$$

Let us choose a representative $\oplus^{+}:|R(A) \times R(A)|^{+} \rightarrow|R(A)|^{+}$for the homotopy class obtained from $\oplus$ by functoriality of the geometric realization and the plusconstruction. Furthermore, let

$$
k^{-1}:|R(A)|^{+} \times|R(A)|^{+} \rightarrow|R(A) \times R(A)|^{+}
$$


denote some homotopy inverse of the homotopy equivalence given by the projection onto each factor. We then define the sum on $|R(A)|^{+}$as the composition

$$
+=\oplus^{+} \circ k^{-1}:|R(A)|^{+} \times|R(A)|^{+} \rightarrow|R(A)|^{+} .
$$

This will be the composition in our commutative $H$-group structure on $|R(A)|^{+}$. The neutral element will be given by the constant map 1: $\Delta^{n} \rightarrow \operatorname{GL}(A)$.

Now, to each injection $u: \mathbb{N} \rightarrow \mathbb{N}$ there is a group homomorphism $u: \operatorname{GL}(A) \rightarrow$ GL $(A)$ defined by

$$
u(g)_{i j}= \begin{cases}g_{k l} & \text { for } i=u(k), j=u(l), \\ \delta_{i j} & \text { elsewhere. }\end{cases}
$$

We extend this construction to a pointwise version, associating a simplicial map

$$
u: R(A) \rightarrow R(A), \quad \sigma \mapsto(t \mapsto u(\sigma(t))),
$$

to each injective map $u: \mathbb{N} \rightarrow \mathbb{N}$. We let $u^{+}:|R(A)|^{+} \rightarrow|R(A)|^{+}$denote a representative of the homotopy class obtained from $u$ by functoriality. The above constructions also applies to the case of the fiber $F(A)$.

Lemma 3.9. For each elementary matrix $g \in E(A)$ the simplicial maps

$$
\operatorname{Ad}_{g}: R(A) \rightarrow R(A) \text { and } \operatorname{Ad}_{g}: F(A) \rightarrow F(A)
$$

given by $\sigma \mapsto g \sigma g^{-1}$ are homotopic to the identity.

Proof. We will only consider the fiber $F(A)$. Let $g \in E(A)$ and let $\gamma \in F(A)_{1}$ satisfy $\gamma\left(v_{0}\right)=1$ and $\gamma\left(v_{1}\right)=g$. In degree $n \geq 1$, a simplicial homotopy between conjugation by our elementary matrix $g \in E(A)$ and the identity is then given by

$$
h_{i}(\sigma)=\left(s_{n} \ldots s_{i+1} s_{i-1} \ldots s_{0}\right)(\gamma) \cdot s_{i}(\sigma), \quad i \in\{0, \ldots, n\} .
$$

Notice that the "extra" factor in the expression (3.1) for the zeroth face operator ensures us that

$$
d_{0}\left(h_{0}(\sigma)\right)=d_{0}\left(s_{n} \ldots s_{1}(\gamma)\right) \gamma\left(v_{1}\right) d_{0}\left(s_{0}(\sigma)\right) \gamma\left(v_{1}\right)^{-1}=g \sigma g^{-1} .
$$

Corollary 3.10. For each injection $u: \mathbb{N} \rightarrow \mathbb{N}$ the associated simplicial maps

$$
u: R(A) \rightarrow R(A) \text { and } u: F(A) \rightarrow F(A)
$$

induce the identity homomorphisms

$$
u_{*}=\mathrm{Id}: H_{*}(R(A)) \rightarrow H_{*}(R(A)) \quad \text { and } \quad u_{*}=\mathrm{Id}: H_{*}(F(A)) \rightarrow H_{*}(F(A))
$$

on homology. 
Proof. With Lemma 3.9 in hand, the proof becomes similar to the proof of [32], Lemma 1.3.

The results obtained in Section 3.1 together with the classical Whitehead theorem now allow us to show that the monoid of injections $u: \mathbb{N} \rightarrow \mathbb{N}$ acts on $|R(A)|^{+}$by homotopy equivalences.

Theorem 3.11. For each injection $u: \mathbb{N} \rightarrow \mathbb{N}$ the induced map

$$
u^{+}:|R(A)|^{+} \rightarrow|R(A)|^{+}
$$

is a homotopy equivalence.

Proof. We show that

$$
u^{+}:|R(A)|^{+} \rightarrow|R(A)|^{+}
$$

is a weak equivalence and refer to Whitehead's theorem.

For $n=1$ we note that $\pi_{1}\left(|R(A)|^{+}\right) \cong H_{1}(R(A))$, so $\pi_{1}\left(u^{+}\right): \pi_{1}\left(|R(A)|^{+}\right) \rightarrow$ $\pi_{1}\left(|R(A)|^{+}\right)$is an isomorphism by Corollary 3.10 .

For $n \geq 2$ we note that $\pi_{n}\left(|F(A)|^{+}\right) \cong \pi_{n}\left(|R(A)|^{+}\right)$by Corollary 3.7. Since the space $|F(A)|^{+}$is simply connected, we will only need to show that $u^{+}:|F(A)|^{+} \rightarrow$ $|F(A)|^{+}$induces an isomorphism in homology. However, this is a consequence of Corollary 3.10.

The commutative $H$-group properties of the sum $+:|R(A)|^{+} \times|R(A)|^{+} \rightarrow$ $|R(A)|^{+}$and the neutral element $1 \in|R(A)|^{+}$can now be obtained by a rephrasing of the arguments in [20], Section 1.2.

Corollary 3.12. For each injection $u: \mathbb{N} \rightarrow \mathbb{N}$ the induced map

$$
u^{+}:|R(A)|^{+} \rightarrow|R(A)|^{+}
$$

is homotopic to the identity.

Proof. This is a consequence of the Grothendieck group of the monoid of injections $u: \mathbb{N} \rightarrow \mathbb{N}$ being trivial; see [20], Lemma 1.2.8.

Theorem 3.13. The application $+:|R(A)|^{+} \times|R(A)|^{+} \rightarrow|R(A)|^{+}$and the neutral element $1 \in|R(A)|^{+}$define a commutative $H$-group structure on $|R(A)|^{+}$.

Proof. That the sum and the neutral element defines a homotopy associative and homotopy commutative $H$-space structure follows from Corollary 3.12 since the appropriate maps are homotopic up to composition with some $u^{+}:|R(A)|^{+} \rightarrow$ $|R(A)|^{+}$. The existence of a homotopy inverse is automatic since we are working exclusively with connected CW-complexes, [28], Theorem 3.4. 
We end this section by considering the relationship between the $H$-group structure on $|R(A)|^{+}$and the $H$-group structure on $\operatorname{BGL}(A)^{+}$. Let $\theta^{+}:|R(A)|^{+} \rightarrow$ $\operatorname{BGL}(A)^{+}$denote a representative of the homotopy class induced by the simplicial map

$$
\theta: R(A) \rightarrow \operatorname{BGL}(A), \quad \theta(\sigma)=\left(\sigma\left(v_{1}\right)^{-1}, \sigma\left(v_{1}\right) \sigma\left(v_{2}\right)^{-1}, \ldots, \sigma\left(v_{n-1}\right) \sigma\left(v_{n}\right)^{-1}\right) .
$$

Theorem 3.14. The map $\theta^{+}:|R(A)|^{+} \rightarrow \operatorname{BGL}(A)^{+}$is an $H$-map.

Proof. This is essentially a matter of checking that the simplicial maps given by

$$
\theta \circ \oplus \text { and } \oplus \circ(\theta \times \theta): R(A) \times R(A) \rightarrow \operatorname{BGL}(A)
$$

coincide.

3.3. Construction of the product in relative K-theory. In this section we show that a pointwise version of the exterior Loday product, determines a multiplicative structure on the relative $K$-groups. The exposition will follow [20, Section 2.1], Section 2.1, closely.

Let $A$ and $B$ be unital Banach algebras.

Let $p, q \in\{3,4, \ldots\}$ be fixed and let $\varphi: A^{p} \otimes_{\mathbb{Z}} B^{q} \rightarrow\left(A \otimes_{\mathbb{Z}} B\right)^{p q}$ denote some isomorphism of $\left(A \otimes_{\mathbb{Z}} B\right)$-bimodules. As in Section 2.1 we have a corresponding map of simplicial sets

$$
\hat{\otimes}_{\varphi}: R_{p}(A) \times R_{q}(B) \rightarrow R_{p q}(A \hat{\otimes} B) .
$$

We choose some $\hat{\otimes}_{\varphi}^{+}:\left|R_{p}(A) \times R_{q}(B)\right|^{+} \rightarrow\left|R_{p q}(A \hat{\otimes} B)\right|^{+}$which represents the homotopy class obtained from $\hat{\otimes}_{\varphi}$ by functoriality. Furthermore, let

$$
k^{-1}:\left|R_{p}(A)\right|^{+} \times\left|R_{q}(B)\right|^{+} \rightarrow\left|R_{p}(A) \times R_{q}(B)\right|^{+}
$$

denote some homotopy inverse to the map given by the projection onto each factor. We then define the tensor product

$$
\hat{\otimes}_{p, q}^{+}=i^{+} \circ \hat{\otimes}_{\varphi}^{+} \circ k^{-1}:\left|R_{p}(A)\right|^{+} \times\left|R_{q}(B)\right|^{+} \rightarrow|R(A \hat{\otimes} B)|^{+} .
$$

Here the map $i^{+}:\left|R_{p q}(A \hat{\otimes} B)\right|^{+} \rightarrow|R(A \hat{\otimes} B)|^{+}$is a representative of the homotopy class determined by the inclusion $i: R_{p q}(A \hat{\otimes} B) \rightarrow R(A \hat{\otimes} B)$.

Following the argumentation of Theorem 3.13 we see that the tensor product only depends on the choice of isomorphism $\varphi: A^{p} \otimes_{\mathbb{Z}} B^{q} \rightarrow\left(A \otimes_{\mathbb{Z}} B\right)^{p q}$ of $\left(A \otimes_{\mathbb{Z}} B\right)$ bimodules up to homotopy. Furthermore, it is natural in $A$ and $B$, bilinear, associative and commutative up to homotopy. The last assertion means that the diagram

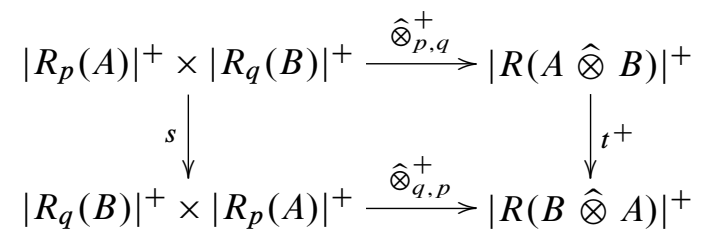


commutes up to homotopy. Both the map $s:\left|R_{p}(A)\right|^{+} \times\left|R_{q}(B)\right|^{+} \rightarrow\left|R_{q}(B)\right|^{+} \times$ $\left|R_{p}(A)\right|^{+}$and the continuous algebra homomorphism $t: A \hat{\otimes} B \rightarrow B \hat{\otimes} A$ change the order of the factors. See also [20], Section 2.1.2.

Now, in order to get a map which descends to the smash product and which behaves well when $p$ and $q$ tend to infinity we define

$$
\begin{gathered}
\gamma_{p, q}^{\mathrm{rel}}:\left|R_{p}(A)\right|^{+} \times\left|R_{q}(B)\right|^{+} \rightarrow|R(A \hat{\otimes} B)|^{+}, \\
(x, y) \mapsto x \hat{\otimes}_{p, q}^{+} y-x \hat{\otimes}_{p, q}^{+} 1_{q}-1_{p} \hat{\otimes}_{p, q}^{+} y .
\end{gathered}
$$

Here the minus sign comes from the commutative $H$-group structure on $|R(A \hat{\otimes} B)|^{+}$ defined in Section 3.2. The elements $1_{p} \in\left|R_{p}(A)\right|^{+}$and $1_{q} \in\left|R_{q}(B)\right|^{+}$are given by the constant maps $1_{p}: \Delta^{n} \rightarrow \mathrm{GL}_{p}(A)$ and $1_{q}: \Delta^{n} \rightarrow \mathrm{GL}_{q}(B)$.

It is immediate that the restriction $\gamma_{p, q}^{\text {rel }}:\left|R_{p}(A)\right|^{+} \vee\left|R_{q}(B)\right|^{+} \rightarrow|R(A \hat{\otimes} B)|^{+}$ is homotopically trivial. Since $|R(A \hat{\otimes} B)|^{+}$is an $H$-group, we thus get a map

$$
\hat{\gamma}_{p, q}^{\text {rel }}:\left|R_{p}(A)\right|^{+} \wedge\left|R_{q}(B)\right|^{+} \rightarrow|R(A \hat{\otimes} B)|^{+}
$$

on the smash product, which is unique up to homotopy and which makes the maps

$$
\hat{\gamma}_{p, q}^{\text {rel }} \circ \pi \quad \text { and } \quad \gamma_{p, q}^{\text {rel }}:\left|R_{p}(A)\right|^{+} \times\left|R_{q}(B)\right|^{+} \rightarrow|R(A \hat{\otimes} B)|^{+}
$$

homotopic. Here $\pi:\left|R_{p}(A)\right|^{+} \times\left|R_{q}(B)\right|^{+} \rightarrow\left|R_{p}(A)\right|^{+} \wedge\left|R_{q}(B)\right|^{+}$denotes the quotient map.

The argumentation presented in [20], p. 333-335, now ensures the existence of a continuous map

$$
\hat{\gamma}^{\mathrm{rel}}:|R(A)|^{+} \wedge|R(B)|^{+} \rightarrow|R(A \hat{\otimes} B)|^{+},
$$

which is natural in $A$ and $B$, bilinear, associative and commutative up to weak homotopies. Furthermore, for any $p, q \in\{3,4, \ldots\}$ the maps

$$
\hat{\gamma}_{p, q}^{\mathrm{rel}} \quad \text { and } \quad \hat{\gamma}^{\mathrm{rel}} \circ\left(i^{+} \wedge i^{+}\right):\left|R_{p}(A)\right|^{+} \wedge\left|R_{q}(B)\right|^{+} \rightarrow|R(A \hat{\otimes} B)|^{+}
$$

agree up to weak homotopy. This enables us to make the following definition.

Definition 3.15. By the exterior product in relative $K$-theory we understand the map

$$
*^{\mathrm{rel}}: K_{n}^{\mathrm{rel}}(A) \times K_{m}^{\mathrm{rel}}(B) \rightarrow K_{n+m}^{\mathrm{rel}}(A \hat{\otimes} B)
$$

given by the formula

$$
[f] *^{\text {rel }}[g]=\left[\hat{\gamma}^{\text {rel }} \circ(f \wedge g)\right]
$$

for each $[f] \in \pi_{n}\left(|R(A)|^{+}\right)$and $[g] \in \pi_{m}\left(|R(B)|^{+}\right)$.

The naturality, bilinearity, associativity and commutativity up to weak homotopies of the map $\hat{\gamma}^{\text {rel }}:|R(A)|^{+} \wedge|R(B)|^{+} \rightarrow|R(A \hat{\otimes} B)|^{+}$imply the corresponding properties for the exterior product. 
Theorem 3.16. The exterior product in relative $K$-theory

$$
*^{\mathrm{rel}}: K_{n}^{\mathrm{rel}}(A) \times K_{m}^{\mathrm{rel}}(B) \rightarrow K_{n+m}^{\mathrm{rel}}(A \hat{\otimes} B)
$$

is natural, bilinear and associative. It is also graded commutative in the sense that

$$
y *^{\mathrm{rel}} x=(-1)^{n m} t_{*}\left(x *^{\mathrm{rel}} y\right) \text { for all } x \in K_{n}^{\mathrm{rel}}(A), y \in K_{m}^{\mathrm{rel}}(B) .
$$

Here $_{*}: K_{n+m}^{\mathrm{rel}}(A \hat{\otimes} B) \rightarrow K_{n+m}^{\mathrm{rel}}(B \hat{\otimes} A)$ is induced by the "flip" homomorphism $t: A \hat{\otimes} B \rightarrow B \hat{\otimes} A$.

In the case where the unital Banach algebra $A$ is commutative we get an interior product

$$
*^{\mathrm{rel}}: K_{n}^{\mathrm{rel}}(A) \times K_{m}^{\mathrm{rel}}(A) \rightarrow K_{n+m}^{\mathrm{rel}}(A)
$$

given by composition of the exterior product with the map induced by the continuous unital algebra homomorphism $\nabla: A \hat{\otimes} A \rightarrow A, a_{1} \otimes a_{2} \mapsto a_{1} \cdot a_{2}$. We are thus able to equip the direct sum of relative $K$-groups $\bigoplus_{k=1}^{\infty} K_{k}^{\text {rel }}(A)$ with the structure of a graded commutative ring. See also [20], Theorem 2.1.12.

3.4. Relations with the Loday product. Our task is now to compare the exterior product of Loday in algebraic K-theory with the exterior product in relative $\mathrm{K}$-theory.

Let $A$ and $B$ be unital Banach algebras. Recall that the Loday product

$$
*: K_{n}(A) \times K_{m}(B) \rightarrow K_{n+m}\left(A \otimes_{\mathbb{Z}} B\right)
$$

is uniquely determined by the continuous maps

$$
\begin{gathered}
\gamma_{p, q}: \mathrm{BGL}_{p}(A)^{+} \times \mathrm{BGL}_{q}(B)^{+} \rightarrow \operatorname{BGL}\left(A \otimes_{\mathbb{Z}} B\right)^{+}, \\
(x, y) \mapsto x \otimes_{p, q}^{+} y-1_{p} \otimes_{p, q}^{+} y-x \otimes_{p, q}^{+} 1_{q} .
\end{gathered}
$$

Here the tensor product $\otimes_{p, q}^{+}: \operatorname{BGL}_{p}(A)^{+} \times \mathrm{BGL}_{q}(B)^{+} \rightarrow \operatorname{BGL}\left(A \otimes_{\mathbb{Z}} B\right)^{+}$is induced by the group homomorphism

$$
\otimes_{\varphi}: \mathrm{GL}_{p}(A) \times \mathrm{GL}_{q}(B) \rightarrow \mathrm{GL}_{p q}\left(A \otimes_{\mathbb{Z}} B\right) \subseteq \mathrm{GL}\left(A \otimes_{\mathbb{Z}} B\right)
$$

associated with an isomorphism $\varphi: A^{p} \otimes_{\mathbb{Z}} B^{q} \rightarrow\left(A \otimes_{\mathbb{Z}} B\right)^{p q}$ of $\left(A \otimes_{\mathbb{Z}} B\right)$ bimodules. The additive compositions come from the commutative $H$-group structure on $\mathrm{BGL}\left(A \otimes_{\mathbb{Z}} B\right)^{+},[20]$.

Definition 3.17. By the completed Loday product in algebraic K-theory we will understand the composition

$$
\hat{*}=\iota_{*} \circ *: K_{n}(A) \times K_{m}(B) \rightarrow K_{n+m}(A \hat{\otimes} B)
$$

of the Loday product and the map induced by the canonical unital ring homomorphism $\iota: A \otimes \mathbb{Z} B \rightarrow A \hat{\otimes} B$. 
We can then show that the homomorphism $\theta: K_{n}^{\mathrm{rel}}(A) \rightarrow K_{n}(A)$ respects the exterior product structures.

Theorem 3.18. For each $x \in K_{n}^{\mathrm{rel}}(A)$ and each $y \in K_{m}^{\mathrm{rel}}(B)$ we have the equality

$$
\theta\left(x *^{\mathrm{rel}} y\right)=\theta(x) \hat{*} \theta(y)
$$

in $K_{n+m}(A \hat{\otimes} B)$. In particular, the map $\theta: \bigoplus_{k \geq 1} K_{k}^{\mathrm{rel}}(A) \rightarrow \bigoplus_{k \geq 1} K_{k}(A)$ is a homomorphism of graded commutative rings whenever $A$ is a commutative, unital Banach algebra.

Proof. Let $p, q \in\{3,4, \ldots\}$ and let $\varphi: A^{p} \otimes_{\mathbb{Z}} B^{q} \rightarrow\left(A \otimes_{\mathbb{Z}} B\right)^{p q}$ denote some isomorphism of $(A \otimes \mathbb{Z} B)$-bimodules. At the level of simplicial sets we then have the equality

$$
\theta\left(\sigma \hat{\otimes}_{\varphi} \tau\right)=\iota\left(\theta(\sigma) \otimes_{\varphi} \theta(\tau)\right) \quad \text { for all } \sigma \in R_{p}(A)_{n}, \tau \in R_{q}(B)_{n} .
$$

This shows that the maps

$\theta^{+} \circ \hat{\otimes}_{p, q}^{+}$and $\iota^{+} \circ \otimes_{p, q}^{+} \circ\left(\theta^{+} \times \theta^{+}\right):\left|R_{p}(A)\right|^{+} \times\left|R_{q}(B)\right|^{+} \rightarrow \operatorname{BGL}(A \hat{\otimes} B)^{+}$ are homotopic. By Theorem 3.14 the map $\theta^{+}:|R(A \hat{\otimes} B)|^{+} \rightarrow \operatorname{BGL}(A \hat{\otimes} B)^{+}$ respects the $H$-group structures up to homotopy, so the maps

$\theta^{+} \circ \gamma_{p, q}^{\mathrm{rel}}$ and $\iota^{+} \circ \gamma_{p, q} \circ\left(\theta^{+} \times \theta^{+}\right):\left|R_{p}(A)\right|^{+} \times\left|R_{q}(B)\right|^{+} \rightarrow \operatorname{BGL}(A \hat{\otimes} B)^{+}$ are homotopic. The desired result now follows by uniqueness of the involved constructions.

\section{On the multiplicative properties of the relative Chern character}

Let $A$ be a unital Banach algebra. Let us start by recalling the construction of the relative Chern character as introduced by A. Connes and M. Karoubi, [9], [17]. By definition, the relative Chern character is obtained as the composition of four maps

$$
\operatorname{ch}^{\mathrm{rel}}: K_{n}^{\mathrm{rel}}(A) \rightarrow \mathrm{HC}_{n-1}(A), \quad \operatorname{ch}^{\mathrm{rel}}=\mathrm{TR} \circ \varepsilon \circ L \circ h_{n} .
$$

We will give a brief description of each of the maps.

The first map is the Hurewicz homomorphism associated with the pointed topological space $|R(A)|^{+}$,

$$
h_{n}: K_{n}^{\mathrm{rel}}(A)=\pi_{n}\left(|R(A)|^{+}\right) \rightarrow H_{n}\left(|R(A)|^{+}\right) \cong H_{n}(R(A)) .
$$

The second map is the logarithm

$$
L: H_{n}(R(A)) \rightarrow \lim _{p \rightarrow \infty} H_{n}^{\mathrm{Lie}}\left(M_{p}(A)\right),
$$


which is given by the chain map

$$
L: \sigma \mapsto \int_{\Delta^{n}} \frac{\partial \sigma}{\partial t_{1}} \cdot \sigma^{-1} \wedge \cdots \wedge \frac{\partial \sigma}{\partial t_{n}} \cdot \sigma^{-1} d t_{1} \ldots d t_{n} .
$$

Here $\sigma: \Delta^{n} \rightarrow \mathrm{GL}_{p}(A)$ is a smooth function; see [29]. Note that we are working with the continuous Lie algebra complex $\left(\Lambda_{*}(A), \delta\right)$, thus in each degree we have a Banach space, $\Lambda_{n}(A)$, in the appropriate quotient norm. This is needed in order for the above integral to make sense; see also Section 2.2.

The third map is the antisymmetrization

$$
\varepsilon: \lim _{p \rightarrow \infty} H_{n}^{\mathrm{Lie}}\left(M_{p}(A)\right) \rightarrow \lim _{p \rightarrow \infty} \mathrm{HC}_{n-1}\left(M_{p}(A)\right),
$$

which is given by the continuous map

$$
\varepsilon: x_{0} \wedge x_{1} \wedge \cdots \wedge x_{n-1} \mapsto \sum_{s \in \Sigma_{n-1}} \operatorname{sgn}(s) x_{0} \otimes x_{s(1)} \otimes \cdots \otimes x_{s(n-1)}
$$

Again, we are working with the continuous cyclic complex $\left(C_{n}^{\lambda}(A), b\right)$, thus in each degree we have a Banach space, $C_{n}^{\lambda}(A)$, in the appropriate quotient norm; see [21], [22].

The last map is the generalized trace on continuous cyclic homology

$$
\text { TR: } \lim _{p \rightarrow \infty} \mathrm{HC}_{n-1}\left(M_{p}(A)\right) \rightarrow \mathrm{HC}_{n-1}(A),
$$

see [21] for example.

The relative Chern character fits in the (up to constants) commutative diagram

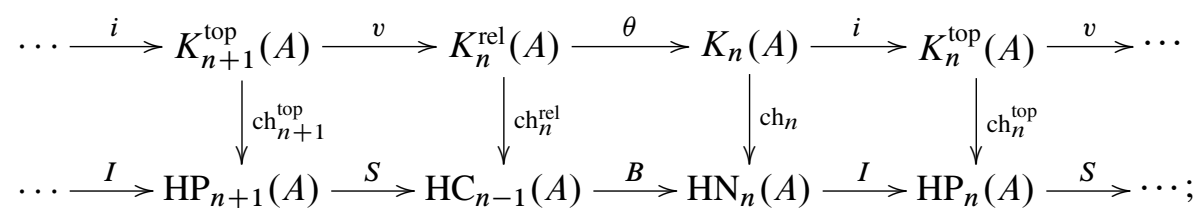

see [18] and [10]. Here the other columns are the continuous versions of the topological Chern character and the continuous version of the algebraic Chern character; see [14], [33] for a definition of the algebraic Chern character. The bottom row is the $S B I$-sequence in continuous cyclic homology. Observe that the relative Chern character defined in this section differs from the one given in [9], [17] by a constant. To be precise, for each $n \in \mathbb{N}$ we have

$$
\mathrm{ch}_{n}^{\mathrm{rel}}=(-1)^{n}(n-1) ! \cdot \underline{\mathrm{ch}}_{n}^{\mathrm{rel}}: K_{n}^{\mathrm{rel}}(A) \rightarrow \mathrm{HC}_{n-1}(A),
$$

where $\mathrm{ch}^{\text {rel }}$ denotes the relative Chern character of [9], Section 3; see also the paper [29]. This alternative definition is necessary for the relative Chern character to respect the product structures. 
4.1. The multiplicative properties of the logarithm. Let $A$ and $B$ be unital Banach algebras. In this section we will show that the logarithm $L: H_{*}\left(R_{p}(A)\right) \rightarrow$ $H_{*}^{\mathrm{Lie}}\left(M_{p}(A)\right)$ respects the product structures on the homology of the simplicial sets $R_{p}(A)$ and the Lie algebra homology of the Banach algebras $M_{p}(A)$. These exterior products were introduced in Section 2.1 and Section 2.2.

For each $n, p \in \mathbb{N}$ and each $j \in\{1, \ldots, n\}$ we define the operator

$$
\Gamma_{j}: C^{\infty}\left(\Delta^{n}, \mathrm{GL}_{p}(A)\right) \rightarrow C^{\infty}\left(\Delta^{n}, M_{p}(A)\right), \quad \Gamma_{j}: \sigma \mapsto \frac{\partial \sigma}{\partial t_{j}} \cdot \sigma^{-1} .
$$

Furthermore we define the wedge product

$$
\begin{gathered}
\gamma: C^{\infty}\left(\Delta^{n}, \operatorname{GL}_{p}(A)\right) \rightarrow C^{\infty}\left(\Delta^{n}, \Lambda_{n}\left(M_{p}(A)\right)\right), \\
\gamma(\sigma)(t)=\Gamma_{1}(\sigma)(t) \wedge \cdots \wedge \Gamma_{n}(\sigma)(t) .
\end{gathered}
$$

Our first task is then to understand the behaviour of $\gamma$ with respect to the exterior shuffle product. This is the content of Lemma 4.1. We will start by introducing some convenient notation.

Let us fix to smooth maps $\sigma: \Delta^{n} \rightarrow \mathrm{GL}_{p}(A)$ and $\tau: \Delta^{m} \rightarrow \mathrm{GL}_{q}(B)$ and let us choose an isomorphism $\varphi: A^{p} \otimes_{\mathbb{Z}} B^{q} \rightarrow\left(A \otimes_{\mathbb{Z}} B\right)^{p q}$ of $\left(A \otimes_{\mathbb{Z}} B\right)$-bimodules. Furthermore we let $(\mu, v) \in \Sigma_{(n, m)}$ be a fixed $(n, m)$-shuffle. To ease the exposition we will assume that $\mu(0)=0$ and that $v(m-1)=n+m-1$.

Let $\left\{A_{0}, A_{1}, \ldots, A_{2 k+1}\right\}$ denote the unique partition of $\{0,1, \ldots, n+m-1\}$ satisfying the conditions

and

$$
\bigcup_{i=0}^{k} A_{2 i}=\operatorname{Im}(\mu), \quad \bigcup_{i=0}^{k} A_{2 i+1}=\operatorname{Im}(v)
$$

$$
i<j \Longrightarrow x<y \quad \text { for all } x \in A_{i}, y \in A_{j} .
$$

Let $r_{i}$ denote the smallest element in $A_{i}$ and let $r_{2 k+2}=n+m$. We associate the composition of degeneracies $s_{A_{i}}=s_{\left(r_{i+1}-1\right)} \circ \cdots \circ s_{r_{i}}$ to each set $A_{i}$ in the partition. We then have the equality

$$
\begin{aligned}
s_{\nu}(\sigma) & \hat{\otimes}_{\varphi} s_{\mu}(\tau) \\
& =s_{A_{2 k+1}} \ldots s_{A_{3}} s_{A_{1}}(\sigma) \hat{\otimes}_{\varphi} s_{A_{2 k}} \ldots s_{A_{2}} s_{A_{0}}(\tau): \Delta^{n+m} \rightarrow \mathrm{GL}_{p q}(A \hat{\otimes} B) .
\end{aligned}
$$

For each $l \in\{0, \ldots, k\}$ we let

$$
E_{l}=\sum_{i=0}^{l}\left|A_{2 i}\right|=\sum_{i=0}^{l}\left(r_{2 i+1}-r_{2 i}\right) \text { and } O_{l}=\sum_{i=0}^{l}\left|A_{2 i+1}\right|=\sum_{i=0}^{l}\left(r_{2 i+2}-r_{2 i+1}\right) \text {. }
$$

We then define the smooth maps

$\omega_{2 l}: \Delta^{n+m} \rightarrow \bigwedge_{\left|A_{2 l}\right|} M_{p q}(A \hat{\otimes} B)$ and $\omega_{2 l+1}: \Delta^{n+m} \rightarrow \bigwedge_{\left|A_{2 l+1}\right|} M_{p q}(A \hat{\otimes} B)$ 
by the wedge products

$$
\omega_{2 l}=s_{v}\left(\Gamma_{E_{l-1}+1}\left(\sigma \hat{\otimes}_{\varphi} 1_{q}\right) \wedge \cdots \wedge \Gamma_{E_{l}}\left(\sigma \hat{\otimes}_{\varphi} 1_{q}\right)\right)
$$

and

$$
\omega_{2 l+1}=s_{\mu}\left(\Gamma_{O_{l-1}+1}\left(1_{p} \hat{\otimes}_{\varphi} \tau\right) \wedge \cdots \wedge \Gamma_{O_{l}}\left(1_{p} \hat{\otimes}_{\varphi} \tau\right)\right)
$$

Finally, let us recall the relations between the degeneracies and the partial differential operators,

$$
\frac{\partial}{\partial t_{j}} \circ s_{i}=\left\{\begin{array}{ll}
s_{i} \circ \frac{\partial}{\partial t_{j-1}} & \text { for } j>i>0, \\
s_{i} \circ \frac{\partial}{\partial t_{j}} & \text { for } j \leq i,
\end{array} \quad \frac{\partial}{\partial t_{j}} \circ s_{0}= \begin{cases}s_{0} \circ \frac{\partial}{\partial t_{j-1}} & \text { for } j>1 \\
0 & \text { for } j=1 .\end{cases}\right.
$$

We can then prove the following technical result.

Lemma 4.1. Let $\sigma: \Delta^{n} \rightarrow \mathrm{GL}_{p}(A)$ and $\tau: \Delta^{m} \rightarrow \mathrm{GL}_{q}(B)$ be a pair of smooth maps. For each $(n, m)$-shuffle $(\mu, v) \in \Sigma_{(n, m)}$ we then have the equality

$$
\gamma\left(s_{\nu}(\sigma) \hat{\otimes}_{\varphi} s_{\mu}(\tau)\right)=\operatorname{sgn}(\mu, \nu) s_{\nu}\left(\gamma\left(\sigma \hat{\otimes}_{\varphi} 1_{q}\right)\right) \wedge s_{\mu}\left(\gamma\left(1_{p} \hat{\otimes}_{\varphi} \tau\right)\right)
$$

between smooth maps $\Delta^{n+m} \rightarrow \bigwedge_{n+m} M_{p q}(A \hat{\otimes} B)$.

Proof. We will assume that $\mu(0)=0$ and that $v(m-1)=n+m-1$. The other cases can be proved using similar arguments.

We start by noting that

$$
\begin{aligned}
\omega_{0} \wedge \omega_{1} \wedge \cdots \wedge \omega_{2 k+1} \\
\quad=\operatorname{sgn}(\mu, \nu)\left(\omega_{0} \wedge \omega_{2} \wedge \cdots \wedge \omega_{2 k}\right) \wedge\left(\omega_{1} \wedge \omega_{3} \wedge \cdots \wedge \omega_{2 k+1}\right) \\
\quad=\operatorname{sgn}(\mu, v) s_{v}\left(\gamma\left(\sigma \widehat{\otimes}_{\varphi} 1_{q}\right)\right) \wedge s_{\mu}\left(\gamma\left(1_{p} \hat{\otimes}_{\varphi} \tau\right)\right) .
\end{aligned}
$$

It is therefore sufficient to prove the identity

$$
\gamma\left(s_{\nu}(\sigma) \hat{\otimes}_{\varphi} s_{\mu}(\tau)\right)=\omega_{0} \wedge \cdots \wedge \omega_{2 k+1} .
$$

We will use induction to show that

$$
\Gamma_{1}\left(s_{\nu}(\sigma) \hat{\otimes}_{\varphi} s_{\mu}(\tau)\right) \wedge \cdots \wedge \Gamma_{r_{i}}\left(s_{\nu}(\sigma) \hat{\otimes}_{\varphi} s_{\mu}(\tau)\right)=\omega_{0} \wedge \cdots \wedge \omega_{i-1}
$$

for each $i \in\{1, \ldots, 2 k+2\}$. Thus, let $j \in\left\{1, \ldots, r_{1}\right\}$. By the identities in (4.1) we get

$$
\begin{aligned}
\frac{\partial}{\partial t_{j}}\left(s_{v}(\sigma) \hat{\otimes}_{\varphi} s_{\mu}(\tau)\right) & =\frac{\partial}{\partial t_{j}}\left(s_{v}(\sigma)\right) \hat{\otimes}_{\varphi} s_{\mu}(\tau)+s_{v}(\sigma) \hat{\otimes}_{\varphi} \frac{\partial}{\partial t_{j}}\left(s_{\mu}(\tau)\right) \\
& =s_{v}\left(\frac{\partial \sigma}{\partial t_{j}}\right) \hat{\otimes}_{\varphi} s_{\mu}(\tau)
\end{aligned}
$$


By consequence we have that

$$
\Gamma_{j}\left(s_{\nu}(\sigma) \hat{\otimes}_{\varphi} s_{\mu}(\tau)\right)=s_{\nu}\left(\Gamma_{j}(\sigma)\right) \hat{\otimes}_{\varphi} 1_{q}=s_{\nu}\left(\Gamma_{j}\left(\sigma \hat{\otimes}_{\varphi} 1_{q}\right)\right),
$$

proving the induction start.

Now suppose that

$$
\Gamma_{1}\left(s_{v}(\sigma) \hat{\otimes}_{\varphi} s_{\mu}(\tau)\right) \wedge \cdots \wedge \Gamma_{r_{i}}\left(s_{v}(\sigma) \hat{\otimes}_{\varphi} s_{\mu}(\tau)\right)=\omega_{0} \wedge \cdots \wedge \omega_{i-1}
$$

for some $i \in\{1, \ldots, 2 k+1\}$. We will only consider the case of $i=2 l$ being even. The odd case can be proved by similar arguments. Thus, let $j \in\left\{r_{2 l}+1, \ldots, r_{2 l+1}\right\}$. By the identities in (4.1) we get

$$
\begin{aligned}
\frac{\partial}{\partial t_{j}}\left(s_{\nu}(\sigma) \hat{\otimes}_{\varphi} s_{\mu}(\tau)\right)= & s_{A_{2 k+1}} \ldots s_{A_{2 l+1}} \frac{\partial}{\partial t_{j}}\left(s_{A_{2 l-1}} \ldots s_{A_{1}}(\sigma)\right) \hat{\otimes}_{\varphi} s_{\mu}(\tau) \\
& +s_{\nu}(\sigma) \hat{\otimes}_{\varphi} s_{A_{2 k}} \ldots s_{A_{2 l+2}} \frac{\partial}{\partial t_{j}}\left(s_{A_{2 l}} \ldots s_{A_{0}}(\tau)\right) \\
= & s_{\nu}\left(\frac{\partial \sigma}{\partial t_{j-O}-O_{l-1}}\right) \hat{\otimes}_{\varphi} s_{\mu}(\tau)+s_{\nu}(\sigma) \hat{\otimes}_{\varphi} s_{\mu}\left(\frac{\partial \tau}{\partial t_{r_{2 l}-E_{l-1}}}\right) .
\end{aligned}
$$

Noting that $r_{2 l}-E_{l-1}=O_{l-1}$ we deduce the identity

$$
\Gamma_{j}\left(s_{\nu}(\sigma) \hat{\otimes}_{\varphi} s_{\mu}(\tau)\right)=s_{v}\left(\Gamma_{j-O_{l-1}}\left(\sigma \hat{\otimes}_{\varphi} 1_{q}\right)\right)+s_{\mu}\left(\Gamma_{O_{l-1}}\left(1_{p} \hat{\otimes}_{\varphi} \tau\right)\right) .
$$

But the term $s_{\mu}\left(\Gamma_{O_{l-1}}\left(1_{p} \hat{\otimes}_{\varphi} \tau\right)\right)$ already appears in the wedge product

$$
\omega_{2 l-1}=s_{\mu}\left(\Gamma_{O_{l-2}+1}\left(1_{p} \hat{\otimes}_{\varphi} \tau\right) \wedge \cdots \wedge \Gamma O_{l-1}\left(1_{p} \hat{\otimes}_{\varphi} \tau\right)\right) .
$$

Using the induction hypothesis we thus get that

$$
\begin{gathered}
\Gamma_{1}\left(s_{\nu}(\sigma) \hat{\otimes}_{\varphi} s_{\mu}(\tau)\right) \wedge \cdots \wedge \Gamma_{r_{2 l+1}}\left(s_{\nu}(\sigma) \hat{\otimes}_{\varphi} s_{\mu}(\tau)\right) \\
=\left(\omega_{0} \wedge \cdots \wedge \omega_{2 l-1}\right) \wedge s_{\nu}\left(\Gamma_{r_{2 l}-O_{(l-1)}+1}\left(\sigma \hat{\otimes}_{\varphi} 1_{q}\right) \wedge \cdots\right. \\
\left.\quad \cdots \wedge \Gamma_{r_{(2 l+1)}-O_{l-1}}\left(\sigma \hat{\otimes}_{\varphi} 1_{q}\right)\right) \\
=\omega_{0} \wedge \cdots \wedge \omega_{2 l},
\end{gathered}
$$

proving the induction step.

To continue further, we will need the following lemma which can be proved by a direct but tedious computation,

Lemma 4.2. For any pair of continuous maps $\alpha: \Delta^{n} \rightarrow A$ and $\beta: \Delta^{m} \rightarrow B$ we have the identity

$$
\begin{aligned}
\sum_{(\mu, \nu) \in \Sigma_{(n, m)}} & \int_{\Delta^{n+m}} s_{\nu}(\alpha) \otimes s_{\mu}(\beta) d t_{1} \ldots d t_{n+m} \\
& =\left(\int_{\Delta^{n}} \alpha d t_{1} \ldots d t_{n}\right) \otimes\left(\int_{\Delta^{m}} \beta d t_{1} \ldots d t_{m}\right)
\end{aligned}
$$

in the unital Banach algebra $A \hat{\otimes} B$. 
Denote by $\phi: M_{p}(A) \hat{\otimes} M_{q}(B) \rightarrow M_{p q}(A \hat{\otimes} B)$ the continuous algebra homomorphism associated with the choice of the isomorphism $\varphi: A^{p} \otimes_{\mathbb{Z}} B^{q} \rightarrow$ $\left(A \otimes_{\mathbb{Z}} B\right)^{p q}$ of $\left(A \otimes_{\mathbb{Z}} B\right)$-bimodules. We are now ready for the main result of this section.

Theorem 4.3. For each pair of smooth maps $\sigma: \Delta^{n} \rightarrow \mathrm{GL}_{p}(A)$ and $\tau: \Delta^{m} \rightarrow$ $\mathrm{GL}_{q}(B)$ we have the equality

$$
L\left(\sigma \times_{\varphi} \tau\right)=\phi_{*}\left(L(\sigma) \wedge^{E} L(\tau)\right)
$$

in $\bigwedge_{n+m} M_{p q}(A \hat{\otimes} B)$.

Proof. Using Lemma 4.1 and Lemma 4.2 we get that

$$
\begin{aligned}
L\left(\sigma \times_{\varphi} \tau\right) & =\sum_{(\mu, v) \in \Sigma_{(n, m)}} \operatorname{sgn}(\mu, \nu) \int_{\Delta^{n+m}} \gamma\left(s_{\nu}(\sigma) \hat{\otimes}_{\varphi} s_{\mu}(\tau)\right) d t_{1} \ldots d t_{n+m} \\
& =\sum_{(\mu, v) \in \Sigma_{(n, m)}} \int_{\Delta^{n+m}} s_{v}\left(\gamma\left(\sigma \hat{\otimes}_{\varphi} 1_{q}\right)\right) \wedge s_{\mu}\left(\gamma\left(1_{p} \hat{\otimes}_{\varphi} \tau\right)\right) d t_{1} \ldots d t_{n+m} \\
& =L\left(\sigma \hat{\otimes}_{\varphi} 1_{q}\right) \wedge L\left(1_{p} \hat{\otimes}_{\varphi} \tau\right) .
\end{aligned}
$$

The desired result now follows by naturality of the logarithm.

4.2. The multiplicative properties of the antisymmetrization. Let $A$ and $B$ be unital Banach algebras. We will now show that the antisymmetrization $\varepsilon: \wedge_{*} A \rightarrow$ $C_{*-1}^{\lambda}(A)$ respects the product structures on the continuous Lie algebra homology and the continuous cyclic homology. The definition of the antisymmetrization is recalled in the beginning of this section and the exterior products considered are defined in Section 2.2 and Section 2.3.

Theorem 4.4. For each $x \in \bigwedge_{n} A$ and each $y \in \bigwedge_{m} B$ we have the equality

$$
\varepsilon\left(x \wedge^{E} y\right)=\varepsilon(x) * \varepsilon(y)
$$

in $C_{n+m-1}^{\lambda}(A \hat{\otimes} B)$.

Proof. Let $x=x_{0} \wedge x_{1} \wedge \cdots \wedge x_{n-1} \in \wedge_{n} A$ and let $y=y_{0} \wedge y_{1} \wedge \cdots \wedge y_{m-1} \in \wedge_{m} B$. For each $i \in\{0,1, \ldots, n+m-1\}$, let

$$
z_{i}= \begin{cases}x_{i} \otimes 1_{B} & \text { for } i \in\{0, \ldots, n-1\}, \\ 1_{A} \otimes y_{i-n} & \text { for } i \in\{n, \ldots, n+m-1\} .\end{cases}
$$

By definition of the exterior wedge product and the antisymmetrization map we get

$$
\varepsilon\left(x \wedge^{E} y\right)=\sum_{s \in \Sigma_{n+m-1}} \operatorname{sgn}(s) z_{0} \otimes z_{s(1)} \otimes \cdots \otimes z_{s(n+m-1)} .
$$


However, using the bijective correspondence

$$
\Sigma_{(n-1, m)} \times\left(\Sigma_{n-1} \times \Sigma_{m}\right) \rightarrow \Sigma_{n+m-1}, \quad(\mu,(\sigma \times \tau)) \mapsto \mu \circ(\sigma \times \tau),
$$

we recognize the right hand side as the exterior Hochschild shuffle product of the elements

$\sum_{\sigma \in \Sigma_{n-1}} \operatorname{sgn}(\sigma) x_{0} \otimes x_{\sigma(1)} \otimes \cdots \otimes x_{\sigma(n-1)} \quad$ and $\quad \sum_{\tau \in \Sigma_{m}} \operatorname{sgn}(\tau) 1_{B} \otimes y_{\tau(0)} \otimes \cdots \otimes y_{\tau(m-1)}$.

We therefore have

$$
\varepsilon\left(x \wedge^{E} y\right)=\varepsilon(x) \times \varepsilon\left(1_{B} \wedge y\right)=\varepsilon(x) \times(s N \varepsilon)(y)=\varepsilon(x) * \varepsilon(y),
$$

proving the desired result.

4.3. The multiplicative properties of the generalized trace. Let $A$ and $B$ be unital Banach algebras. In this section we will show that the generalized trace TR: $C_{*}^{\lambda}\left(M_{p}(A)\right) \rightarrow C_{*}^{\lambda}(A)$ respects the exterior product of degree one in continuous cyclic homology.

Let $\phi: M_{p}(A) \hat{\otimes} M_{q}(B) \rightarrow M_{p q}(A \hat{\otimes} B)$ denote the continuous algebra homomorphism induced by some isomorphism $\varphi: A^{p} \otimes_{\mathbb{Z}} B^{q} \rightarrow\left(A \otimes_{\mathbb{Z}} B\right)^{p q}$ of $\left(A \otimes_{\mathbb{Z}} B\right)$-bimodules.

Theorem 4.5. For each $x \in C_{n}^{\lambda}\left(M_{p}(A)\right)$ and each $y \in C_{m}^{\lambda}\left(M_{q}(B)\right)$ we have the equality

$$
\operatorname{TR}(x) * \operatorname{TR}(y)=\left(\operatorname{TR} \circ \phi_{*}\right)(x * y)
$$

in $C_{n+m+1}^{\lambda}(A \hat{\otimes} B)$.

Proof. Let $u \in M_{p}(\mathbb{C})$ and let $v \in M_{q}(\mathbb{C})$. We start by noticing the identity $\operatorname{Tr}(\phi(u \otimes v))=\operatorname{Tr}(u) \operatorname{Tr}(v)$. Here $\operatorname{Tr}: M_{k}(\mathbb{C}) \rightarrow \mathbb{C}$ denotes the usual trace.

Using the formula of [21], Lemma 1.2.2, for the generalized trace we thus get that

$$
\left(\mathrm{TR} \circ \phi_{*}\right)(x \times(s N)(y))=\operatorname{TR}(x) \times(\operatorname{TR} s N)(y) .
$$

The result of the theorem then follows from the identity $(\operatorname{TR} s N)(y)=(s N \mathrm{TR})(y)$, see [21], Lemma 2.2.8, for example.

4.4. The multiplicative properties of the Hurewicz homomorphism. Let $A$ and $B$ be unital Banach algebras. In this section we will investigate the behaviour of the Hurewicz homomorphism with respect to the exterior product in relative K-theory and the exterior shuffle product on the homology of the simplicial sets $R_{p}(A)$. The exterior product in relative K-theory was constructed in Section 3 and the exterior shuffle product was defined in Section 2.1. 
For each $n \in \mathbb{N}$ denote the class of $1 \in \mathbb{Z}$ under the isomorphism $\mathbb{Z} \cong H_{n}\left(S^{n}\right)$ by $\mathbf{1}_{n} \in H_{n}\left(S^{n}\right)$. Furthermore, we let

$$
\text { sh: } H_{n}\left(S^{n}\right) \otimes_{\mathbb{Z}} H_{m}\left(S^{m}\right) \rightarrow H_{n+m}\left(S^{n} \times S^{m}\right)
$$

denote the shuffle map in singular homology. Let $\pi: S^{n} \times S^{m} \rightarrow S^{n} \wedge S^{m}$ denote the quotient map. We then get the equality

$$
\left(\pi_{*} \circ \mathrm{sh}\right)\left(\mathbf{1}_{n} \otimes \mathbf{1}_{m}\right)=\mathbf{1}_{n+m}
$$

in $H_{n+m}\left(S^{n+m}\right) \cong \mathbb{Z}$. For notational reasons we define

$$
\zeta:=\operatorname{sh}\left(\mathbf{1}_{n} \otimes \mathbf{1}_{m}\right) \in H_{n+m}\left(S^{n} \times S^{m}\right) .
$$

The next lemma is the first step needed in order to express the Hurewicz homomorphism of a product in terms of the Hurewicz homomorphism of the original elements.

Lemma 4.6. Let $p, q \in\{3,4, \ldots\}$. Let $f: S^{n} \rightarrow\left|R_{p}(A)\right|^{+}$and $g: S^{m} \rightarrow$ $\left|R_{q}(B)\right|^{+}$be continuous maps. We then have the equality

$$
\left(f \hat{\otimes}_{p, q}^{+} g\right)_{*}(\zeta)=i_{*}\left(h_{n}(f) \times h_{m}(g)\right)
$$

in $H_{n+m}(R(A \hat{\otimes} B)) \cong H_{n+m}\left(|R(A \hat{\otimes} B)|^{+}\right)$. Here $i: R_{p q}(A \hat{\otimes} B) \rightarrow R(A \hat{\otimes}$ $B)$ denotes the inclusion.

Proof. Let us fix an isomorphism $\varphi: A^{p} \otimes_{\mathbb{Z}} B^{q} \rightarrow\left(A \otimes_{\mathbb{Z}} B\right)^{p q}$ of $\left(A \otimes_{\mathbb{Z}} B\right)$ bimodules.

Up to canonical identifications in homology we get that the compositions

$$
\begin{aligned}
\left(\hat{\otimes}_{p, q}^{+}\right)_{*} & \circ \operatorname{sh}: H_{n}\left(\left|R_{p}(A)\right|^{+}\right) \otimes_{\mathbb{Z}} H_{m}\left(\left|R_{q}(B)\right|^{+}\right) \\
& \rightarrow H_{n+m}\left(\left|R_{p}(A)\right|^{+} \times\left|R_{q}(B)\right|^{+}\right) \rightarrow H_{n+m}\left(|R(A \hat{\otimes} B)|^{+}\right)
\end{aligned}
$$

and

$$
\begin{aligned}
i_{*} \circ\left(\hat{\otimes}_{\varphi}\right)_{*} \circ \mathrm{sh}: H_{n}\left(R_{p}(A)\right) \otimes_{\mathbb{Z}} H_{m}\left(R_{q}(B)\right) \\
\quad \rightarrow H_{n+m}\left(R_{p}(A) \times R_{q}(B)\right) \rightarrow H_{n+m}(R(A \hat{\otimes} B))
\end{aligned}
$$

coincide; see Section 2.1 and Section 3.3.

By definition of the exterior shuffle product and the Hurewicz homomorphism we thus have

$$
i_{*}\left(h_{n}(f) \times h_{m}(g)\right)=\left(\left(\hat{\otimes}_{p, q}^{+}\right)_{*} \circ \operatorname{sh}\right)\left(f_{*}\left(\mathbf{1}_{n}\right) \otimes g_{*}\left(\mathbf{1}_{m}\right)\right)=\left(\left(\hat{\otimes}_{p, q}^{+}\right)_{*} \circ(f \times g)_{*}\right)(\zeta)
$$

proving the lemma.

The combination of the next lemma and Lemma 4.6 entails that the Hurewicz homomorphism respects the product structures in an appropriate sense. 
Lemma 4.7. Suppose that the elements $x \in K_{n}^{\mathrm{rel}}(A)$ and $y \in K_{m}^{\mathrm{rel}}(B)$ are represented by the continuous maps

$$
f: S^{n} \rightarrow\left|R_{p}(A)\right|^{+} \subseteq|R(A)|^{+} \text {and } g: S^{m} \rightarrow\left|R_{q}(B)\right|^{+} \subseteq|R(B)|^{+},
$$

respectively. We then have the equality

$$
h_{n+m}\left(x *^{\text {rel }} y\right)=\left(\gamma_{p, q}^{\mathrm{rel}} \circ(f \times g)\right)_{*}(\zeta)
$$

in $H_{n+m}\left(|R(A \hat{\otimes} B)|^{+}\right)$. Here $\gamma_{p, q}^{\text {rel }}:\left|R_{p}(A)\right|^{+} \times\left|R_{q}(B)\right|^{+} \rightarrow|R(A \hat{\otimes} B)|^{+}$ denotes the product map constructed in Section 3.3.

Proof. By definition, the product $x *^{\text {rel }} y \in K_{n+m}^{\text {rel }}(A \hat{\otimes} B)$ is represented by the map

$$
\hat{\gamma}_{p, q}^{\text {rel }} \circ(f \wedge g): S^{n} \wedge S^{m} \rightarrow|R(A \hat{\otimes} B)|^{+} .
$$

Using (4.2) we thus get that the Hurewicz homomorphism of the product is given by

$$
\begin{aligned}
h_{n+m}\left(x *^{\text {rel }} y\right) & =\left(\hat{\gamma}_{p, q}^{\mathrm{rel}} \circ(f \wedge g)\right)_{*}\left(\mathbf{1}_{n+m}\right) \\
& =\left(\hat{\gamma}_{p, q}^{\mathrm{rel}} \circ(f \wedge g) \circ \pi\right)_{*}(\zeta) \\
& =\left(\hat{\gamma}_{p, q}^{\mathrm{rel}} \circ \pi \circ(f \times g)\right)_{*}(\zeta) .
\end{aligned}
$$

The result of the lemma now follows by noting that the maps

$$
\hat{\gamma}_{p, q}^{\text {rel }} \circ \pi \quad \text { and } \quad \gamma_{p, q}^{\text {rel }}:\left|R_{p}(A)\right|^{+} \times\left|R_{q}(B)\right|^{+} \rightarrow|R(A \hat{\otimes} B)|^{+}
$$

are homotopic. See Section 3.3.

4.5. The relative Chern character respects the exterior products. Let $A$ and $B$ be unital Banach algebras. We are now ready to prove the main result of this part of the paper: The counterpart in continuous cyclic homology of the exterior product in relative K-theory is given by the exterior product of degree one. The relevant multiplicative structures are described in Section 3.3 and Section 2.3.

Let $+: \mathrm{HC}_{*}(A) \oplus \mathrm{HC}_{*}(A) \rightarrow \mathrm{HC}_{*}(A)$ denote the addition on the continuous cyclic homology groups. Let $\pi: H_{*}(R(A) \times R(A)) \rightarrow H_{*}(R(A)) \oplus H_{*}(R(A))$ denote the map induced by the projection onto each factor. Furthermore, let $\oplus: R(A) \times$ $R(A) \rightarrow R(A)$ denote the pointwise direct sum as introduced in Section 3.2. We will need the following preliminary result on the additive structures.

Lemma 4.8. We have the equality

$$
+\circ((\mathrm{TR} \circ \varepsilon \circ L) \oplus(\mathrm{TR} \circ \varepsilon \circ L)) \circ \pi=\mathrm{TR} \circ \varepsilon \circ L \circ \oplus_{*}
$$

between maps $H_{n}(R(A) \times R(A)) \rightarrow \mathrm{HC}_{n-1}(A)$. 
Proof. The result is a consequence of the naturality of the involved maps and the behaviour of the generalized trace with respect to the direct sum operation.

Using the work accomplished in Sections 4.1, 4.2, 4.3 and 4.4 we are now able to prove the first main theorem of this paper.

Theorem 4.9. For each $x \in K_{n}^{\mathrm{rel}}(A)$ and each $y \in K_{m}^{\mathrm{rel}}(B)$ we have the equality

$$
\operatorname{ch}^{\mathrm{rel}}\left(x * *^{\mathrm{rel}} y\right)=\operatorname{ch}^{\mathrm{rel}}(x) * \operatorname{ch}^{\mathrm{rel}}(y)
$$

in $\mathrm{HC}_{n+m-1}(A \hat{\otimes} B)$.

Proof. Suppose that $x \in K_{n}^{\mathrm{rel}}(A)$ and $y \in K_{m}^{\mathrm{rel}}(B)$ are represented by the maps $f: S^{n} \rightarrow\left|R_{p}(A)\right|^{+} \subseteq|R(A)|^{+}$and $g: S^{m} \rightarrow\left|R_{q}(B)\right|^{+} \subseteq|R(B)|^{+}$, respectively. By Lemma 4.7 we have

$$
\begin{aligned}
\operatorname{ch}^{\mathrm{rel}}\left(x *^{\mathrm{rel}} y\right) & =\left(\mathrm{TR} \circ \varepsilon \circ L \circ h_{n+m}\right)\left(x *^{\mathrm{rel}} y\right) \\
& =(\mathrm{TR} \circ \varepsilon \circ L) \circ\left(\gamma_{p, q}^{\mathrm{rel}} \circ(f \times g)\right)_{*}(\zeta) .
\end{aligned}
$$

However it follows by definition of $\gamma_{p, q}^{\text {rel }}:\left|R_{p}(A)\right|^{+} \times\left|R_{q}(B)\right|^{+} \rightarrow|R(A \hat{\otimes} B)|^{+}$ and by Lemma 4.6 and Lemma 4.8 that

$$
\begin{aligned}
(\mathrm{TR} \circ \varepsilon \circ L) \circ\left(\gamma_{p, q}^{\mathrm{rel}} \circ(f \times g)\right)_{*}(\zeta) & \\
= & (\mathrm{TR} \circ \varepsilon \circ L)\left(h_{n}(f) \times h_{m}(g)\right)+(\mathrm{TR} \circ \varepsilon \circ L)\left(\left(V_{*} \circ i_{*}\right)\left(h_{n}\left(1_{p}\right) \times h_{m}(g)\right)\right) \\
& \quad+(\mathrm{TR} \circ \varepsilon \circ L)\left(\left(V_{*} \circ i_{*}\right)\left(h_{n}(f) \times h_{m}\left(1_{q}\right)\right)\right) .
\end{aligned}
$$

Here $V:|R(A \hat{\otimes} B)|^{+} \rightarrow|R(A \hat{\otimes} B)|^{+}$denotes the homotopy inverse of the $H$-group structure on $|R(A \hat{\otimes} B)|^{+}$, see Section 3.2. But the elements $h_{n}\left(1_{p}\right) \in$ $H_{n}\left(R_{p}(A)\right)$ and $h_{m}\left(1_{q}\right) \in H_{m}\left(R_{q}(B)\right)$ are both trivial so we must have

$$
\operatorname{ch}^{\mathrm{rel}}\left(x *^{\mathrm{rel}} y\right)=(\mathrm{TR} \circ \varepsilon \circ L)\left(h_{n}(f) \times h_{m}(g)\right) .
$$

The conclusion of the theorem now follows from a combination of the results in Theorem 4.3, Theorem 4.4 and Theorem 4.5.

Remark 4.10. It is possible to regard the main result of this section on the multiplicative properties of the relative Chern character from a different point of view. This alternative approach was suggested to us by the referee.

In order to explain the ideas properly we will need to introduce some notation. Let $A_{*}$ denote the simplicial unital Fréchet algebra that in degree $n \in \mathbb{N}_{0}$ consists of the smooth maps from the $n$-simplex $\Delta^{n}$ to $A$,

$$
A_{n}:=C^{\infty}\left(\Delta^{n}, A\right) .
$$


The face and degeneracy operators are the standard ones. We define the Kan complex $\mathrm{KV}^{\text {top }}(A)$ as the diagonal of the bisimplicial set $\mathrm{BGL}\left(A_{*}\right)$. The homotopy groups of $\mathrm{KV}^{\text {top }}(A)$ agree with the topological $K$-groups of the unital Banach algebra $A$.

Now, regarding elements of the general linear group over $A$ as constant smooth maps we get a map of simplicial sets

$$
\pi: \operatorname{BGL}(A) \rightarrow \mathrm{KV}^{\mathrm{top}}(A) .
$$

By the universal property of the plus-construction there is a continuous map

$$
\pi^{+}:|\operatorname{BGL}(A)|^{+} \rightarrow\left|\mathrm{KV}^{\mathrm{top}}(A)\right|,
$$

which is well defined up to homotopy. Let $\widetilde{F}_{\pi^{+}}^{A}$ denote the homotopy fiber of such a representative. It can then be proved that the map $\theta:|R(A)|^{+} \rightarrow|\operatorname{BGL}(A)|^{+}$ factorizes to a homotopy equivalence between $|R(A)|^{+}$and the homotopy fiber $\mathcal{F}_{\pi^{+}}^{A}$. See [17], Proposition 6.17.

The multiplicative structure in relative K-theory which we have introduced in Section 3 could now probably be seen in a different way. The map $\pi^{+}$is going to respect the completed Loday product at the level of spaces. The completed Loday product will therefore induce a map between appropriate homotopy fibers. In particular, there is a continuous map

$$
\hat{\alpha}^{\text {rel }}: \mathscr{F}_{\pi^{+}}^{A} \wedge \mathscr{F}_{\pi^{+}}^{B} \rightarrow \mathscr{F}_{\pi^{+}}^{A \hat{\otimes} B} .
$$

Under the homotopy equivalence $|R(A)|^{+} \cong \mathscr{F}_{\pi^{+}}^{A}$, this map is likely to coincide with the product map

$$
\hat{\gamma}^{\text {rel }}:|R(A)|^{+} \wedge|R(B)|^{+} \rightarrow|R(A \hat{\otimes} B)|^{+}
$$

defined in Section 3.3.

The relative Chern character can be explained differently as well. Let $\mathscr{B}(A)$, $\mathscr{B}^{-}(A)$ and $\mathscr{B}^{\text {per }}(A)$ denote the $(b, B)$-bicomplexes which define the continuous versions of cyclic homology, negative cyclic homology and periodic cyclic homology; see [21] for example. Furthermore, let

$$
\Gamma: \mathrm{Ch}_{n \geq 0} \rightarrow \mathrm{sAb}
$$

denote the covariant functor which by the Dold-Kan correspondence associates a simplicial abelian group to a chain complex. This functor could be defined by one of the explicit constructions in [19]. To ease the notation let us denote the simplicial abelian group $\Gamma\left(\operatorname{Tot}\left(B^{-}(A)\right)\right)$ by $\mathrm{HN}(A)$ and the diagonal of the bisimplicial abelian group $\Gamma\left(\operatorname{Tot}\left(\mathscr{B}^{-}\left(A_{*}\right)\right)\right)$ by $\mathrm{HN}\left(A_{*}\right)$. It can then be proved that there exist continuous maps

$$
\operatorname{ch}^{\text {alg }}:|\operatorname{BGL}(A)|^{+} \rightarrow|\mathrm{HN}(A)| \quad \text { and } \quad \operatorname{ch}^{\text {top }}:\left|\mathrm{KV}^{\text {top }}(A)\right| \rightarrow\left|\mathrm{HN}\left(A_{*}\right)\right| ;
$$


see [24], [33]. In this setup the topological Chern character $\mathrm{ch}^{\text {top }}$ is defined by the same procedure as the algebraic Chern character $\mathrm{ch}^{\text {alg }}$. These maps will therefore make the diagram

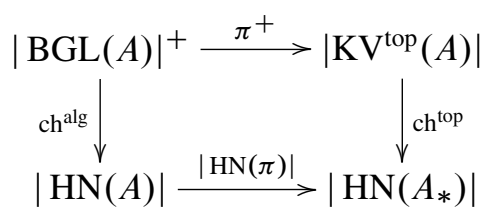

commute up to homotopy. This implies the existence of a map between the homotopy fibers

$$
\mathrm{ch}^{\mathrm{rel}}: \mathscr{F}_{\pi^{+}}^{A} \rightarrow \mathscr{F}_{|\mathrm{HN}(\pi)|}^{A} \cdot
$$

The homotopy groups of the homotopy fiber $\mathcal{F}_{\mid} \mathrm{HN}(\pi) \mid$ identify with the continuous cyclic homology of $A$ with a shift in degree. To be more precise, there is an isomorphism

$$
\pi_{n}\left(\widetilde{F}_{|\mathrm{HN}(\pi)|}\right) \cong \mathrm{HC}_{n-1}(A) .
$$

This is proved in [10], Lemma 4.2.2. After taking homotopy groups we thus get a homomorphism

$$
\operatorname{ch}^{\text {rel }}: K_{n}^{\text {rel }}(A) \rightarrow \mathrm{HC}_{n-1}(A) .
$$

Up to constants this homomorphism agrees with the relative Chern character which we have been discussing in Section 4; see [18], Théorème 4.4.

The multiplicative properties of the relative Chern character could now be investigated by looking at the multiplicative properties of the algebraic Chern character. The completed Hood-Jones product in continuous negative cyclic homology induces a continuous map

$$
\times:|\mathrm{HN}(A)| \wedge|\mathrm{HN}(B)| \rightarrow|\mathrm{HN}(A) \times \mathrm{HN}(B)| \rightarrow|\mathrm{HN}(A \hat{\otimes} B)| .
$$

Using the results of [11], [14], [24] it should then be proved that the diagram

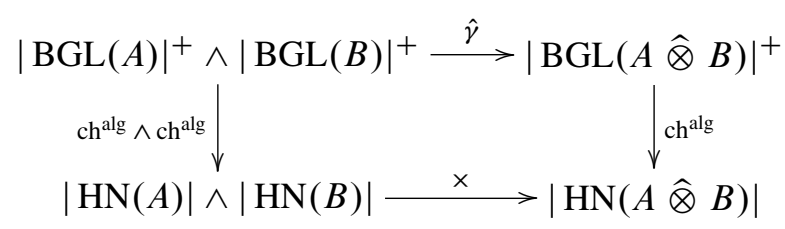

commutes up to homotopy. Since the topological Chern character is just a variant of the algebraic Chern character the corresponding diagram would commute in the topological setting as well. These results entail the commutativity of the homotopy fiber diagram

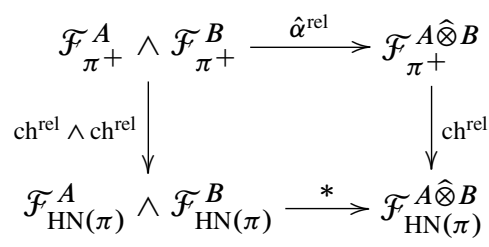


up to homotopy. The bottom row product is given by the completed Hood-Jones product at the homotopy fiber level. After proper identifications this should provide a different proof of Theorem 4.9. We would of course like to thank the referee for this valuable remark.

\section{A calculation of the multiplicative character}

We start this section by briefly recalling the construction of the multiplicative character as given in [9]. See also [16], [27].

Let $n \in \mathbb{N}$ and let $A$ be a unital Banach algebra. Let $(F, H)$ be an $n$-summable Fredholm module over $A$. The parity of the Fredholm module is assumed to be the same as the parity of the dimension, $n-1$. To ease the exposition we will assume that the representation $\pi: A \rightarrow \mathscr{L}(H)$ and the map $a \mapsto[F, \pi(a)] \in \mathscr{L}^{n}(H)$ are both continuous. We will always suppress the representation. Remark that the conditions on continuity are not necessary for the construction of the multiplicative character to work. They are however convenient for our exposition. See [9].

The continuous linear map

$$
\tau_{F}: C_{n-1}^{\lambda}(A) \rightarrow \mathbb{C},\left(a_{0}, \ldots, a_{n-1}\right) \mapsto c_{n-1} \operatorname{Tr}\left(\gamma^{n} F\left[F, a_{0}\right] \ldots\left[F, a_{n-1}\right]\right),
$$

determines a continuous cyclic cocycle and consequently a homomorphism

$$
\tau_{F}: \operatorname{HC}_{n-1}(A) \rightarrow \mathbb{C} .
$$

Here $c_{n-1} \in \mathbb{Q}$ is the rational constant

$$
c_{n-1}= \begin{cases}-\frac{1}{2^{2 m \cdot(m-1) !}} & \text { for } n=2 m, \\ (-1)^{m+1} \frac{m !}{2 \cdot(2 m) !} & \text { for } n=2 m+1,\end{cases}
$$

and $\gamma \in \mathscr{L}(H)$ is the grading operator of the Fredholm module $(F, H)$. The convention is to put $\gamma=\mathrm{Id}$ in the odd case; see [7], [8]. The composition of this index cocycle with the relative Chern character thus yields a homomorphism

$$
\mathcal{A}_{F}:=\tau_{F} \circ \operatorname{ch}_{n}^{\mathrm{rel}}: K_{n}^{\mathrm{rel}}(A) \rightarrow \mathbb{C} .
$$

This is the additive character of the Fredholm module.

The next step in the construction consists of showing that the image of the composition

$$
\tau_{F} \circ \operatorname{ch}_{n}^{\text {rel }} \circ v: K_{n+1}^{\mathrm{top}}(A) \rightarrow \mathbb{C}
$$

is contained in the additive subgroup $(2 \pi i)^{\lceil n / 2\rceil} \mathbb{Z} \subseteq \mathbb{C}$. Here the map $v: K_{n+1}^{\text {top }}(A) \rightarrow$ $K_{n}^{\mathrm{rel}}(A)$ is the boundary map of the long exact sequence (3.2). This is accomplished in [9], Section 4.10. By consequence the additive character descends to a homomorphism

$$
\mathcal{M}_{F}: \operatorname{coker}(v) \cong \operatorname{Im}(\theta) \rightarrow \mathbb{C} /(2 \pi i)^{\lceil n / 2\rceil} \mathbb{Z} .
$$


This is the multiplicative character associated with the $n$-summable Fredholm module $(F, H)$. With some further effort the multiplicative character can be extended to a map on algebraic K-theory, however we will only need the restriction to the subgroup $\operatorname{Im}(\theta) \subseteq K_{n}(A)$ for our calculations. We remark that the rational constants $c_{n-1} \in \mathbb{Q}$ in the definition of the continuous cyclic cocycle (5.1) are chosen in such a way that our definition of the multiplicative character agrees with the one given in [9].

5.1. The relative Chern character of a product of contractions. Let $A$ be a commutative, unital Banach algebra. In this section we will give a concrete formula for the application of the relative Chern character to products of certain elements in relative K-theory. We will make use of the multiplicative properties of the relative Chern character which we investigated in Section 4.

We let

$$
*^{\mathrm{rel}}: K_{k}^{\mathrm{rel}}(A) \times K_{m}^{\mathrm{rel}}(A) \rightarrow K_{k+m}^{\mathrm{rel}}(A)
$$

and

$$
*: \mathrm{HC}_{k-1}(A) \otimes_{\mathbb{C}} \mathrm{HC}_{m-1}(A) \rightarrow \mathrm{HC}_{k+m-1}(A)
$$

denote the (interior) products in relative K-theory and continuous cyclic homology. Note that these products are only available by the commutativity assumption on $A$; see Section 3.3 and Section 2.3.

Furthermore, for each $a \in M_{\infty}(A)$ we let $\gamma_{a} \in R(A)_{1}$ denote the smooth path defined by

$$
\gamma_{a}(t)=e^{-t a} \quad \text { for all } t \in[0,1] .
$$

Theorem 5.1. Let $n \in \mathbb{N}$ and let $a_{0}, \ldots, a_{n-1} \in M_{\infty}(A)$. The relative Chern character of the product

$$
[\gamma]=\left[\gamma_{a_{0}}\right] *^{\mathrm{rel}} \cdots *^{\mathrm{rel}}\left[\gamma_{a_{n-1}}\right] \in K_{n}^{\mathrm{rel}}(A)
$$

is given by

$\operatorname{ch}^{\mathrm{rel}}[\gamma]=(-1)^{n} \sum_{\mu \in \Sigma_{n-1}} \operatorname{sgn}(\mu) \operatorname{TR}\left(a_{0}\right) \otimes \operatorname{TR}\left(a_{\mu(1)}\right) \otimes \cdots \otimes \operatorname{TR}\left(a_{\mu(n-1)}\right) \in \mathrm{HC}_{n-1}(A)$.

Proof. By Theorem 4.9 we have

$$
\operatorname{ch}^{\mathrm{rel}}[\gamma]=\operatorname{ch}^{\mathrm{rel}}\left[\gamma_{a_{0}}\right] * \cdots * \operatorname{ch}^{\mathrm{rel}}\left[\gamma_{a_{n-1}}\right] \in \mathrm{HC}_{n-1}(A) .
$$

Furthermore, the relative Chern character of the individual terms is given by

$$
\operatorname{ch}^{\mathrm{rel}}\left(\left[\gamma_{a}\right]\right)=(\mathrm{TR} \circ \varepsilon \circ L)\left(\left[\gamma_{a}\right]\right)=\operatorname{TR}\left(\int_{0}^{1} \frac{d \gamma_{a}}{d t} \cdot \gamma_{a}^{-1} d t\right)=-\operatorname{TR}(a)
$$

for each $a \in M_{\infty}(A)$. The desired result now follows by definition of the product of degree one in continuous cyclic homology. 
5.2. An evaluation of the multiplicative character on higher Loday products. We are now ready to prove our concrete formula for the application of the multiplicative character to higher Loday products. This will accomplish the main purpose of the paper.

Let $(F, H)$ be an $n$-summable Fredholm module over a commutative, unital Banach algebra $A$. We will suppose that the representation $\pi: A \rightarrow \mathscr{L}(H)$ and the linear map $a \mapsto[F, \pi(a)] \in \mathscr{L}^{n}(H)$ are continuous. We refer to the beginning of Section 5 for a brief reminder on the construction of the multiplicative character.

Theorem 5.2. Let $a_{0}, \ldots, a_{n-1} \in M_{\infty}(A)$. The multiplicative character of the Loday product $\left[e^{a_{0}}\right] * \cdots *\left[e^{a_{n-1}}\right] \in K_{n}(A)$ is then given by

$$
\begin{aligned}
& \mathcal{M}_{F}\left(\left[e^{a_{0}}\right] * \cdots *\left[e^{a_{n-1}}\right]\right) \\
& =(-1)^{n} \sum_{\mu \in \Sigma_{n-1}} \operatorname{sgn}(\mu)\left(q \circ \tau_{F}\right)\left(\operatorname{TR}\left(b_{0}\right) \otimes \operatorname{TR}\left(b_{\mu(1)}\right) \otimes \cdots \otimes \operatorname{TR}\left(b_{\mu(n-1)}\right)\right) \\
& \quad \in \mathbb{C} /(2 \pi i)^{\lceil n / 2\rceil} \mathbb{Z} .
\end{aligned}
$$

Here $q: \mathbb{C} \rightarrow \mathbb{C} /(2 \pi i)^{\lceil n / 2\rceil} \mathbb{Z}$ is the quotient map and $b_{0}, \ldots, b_{n-1} \in M_{\infty}(A)$ are any elements with

$$
e^{b_{i}}=e^{a_{i}} \quad \text { for all } i \in\{0, \ldots, n-1\} .
$$

Proof. Let $b_{0}, \ldots, b_{n-1} \in M_{\infty}(A)$ be any logarithms of $e^{a_{0}}, \ldots, e^{a_{n-1}} \in \operatorname{GL}(A)$. For each $i \in\{0, \ldots, n-1\}$ we let $\gamma_{b_{i}} \in R(A)_{1}$ denote the smooth path given by

$$
\gamma_{b_{i}}: t \mapsto e^{-t b_{i}}
$$

We then have

$$
\theta\left(\left[\gamma_{b_{i}}\right]\right)=\left[\gamma_{b_{i}}(1)^{-1}\right]=\left[e^{b_{i}}\right]=\left[e^{a_{i}}\right] .
$$

By Theorem 3.18 the map $\theta: \bigoplus_{k=1}^{\infty} K_{k}^{\text {rel }}(A) \rightarrow \bigoplus_{k=1}^{\infty} K_{k}(A)$ is a homomorphism of graded rings, so we get that

$$
\theta\left(\left[\gamma_{b_{0}}\right] *^{\mathrm{rel}} \cdots *^{\mathrm{rel}}\left[\gamma_{b_{n-1}}\right]\right)=\left[e^{a_{0}}\right] * \cdots *\left[e^{a_{n-1}}\right] .
$$

By definition of the multiplicative character we then have

$$
\mathcal{M}_{F}\left(\left[e^{a_{0}}\right] * \cdots *\left[e^{a_{n-1}}\right]\right)=\left(q \circ \tau_{F} \circ \mathrm{ch}^{\mathrm{rel}}\right)\left(\left[\gamma_{b_{0}}\right] * *^{\mathrm{rel}} \cdots * *^{\mathrm{rel}}\left[\gamma_{b_{n-1}}\right]\right) \in \mathbb{C} /(2 \pi i)^{\lceil n / 2\rceil} \mathbb{Z} .
$$

But it follows from Theorem 5.1 that the right hand side is given by

$$
\begin{aligned}
& \left(q \circ \tau_{F} \circ \mathrm{ch}^{\mathrm{rel}}\right)\left(\left[\gamma_{b_{0}}\right] *^{\mathrm{rel}} \cdots *^{\mathrm{rel}}\left[\gamma_{b_{n-1}}\right]\right) \\
& \quad=(-1)^{n} \sum_{\mu \in \Sigma_{n-1}} \operatorname{sgn}(\mu)\left(q \circ \tau_{F}\right)\left(\operatorname{TR}\left(b_{0}\right) \otimes \operatorname{TR}\left(b_{\mu(1)}\right) \otimes \cdots \otimes \operatorname{TR}\left(b_{\mu(n-1)}\right)\right),
\end{aligned}
$$

proving the desired result. 
Corollary 5.3. For any commutative unital Banach algebra the multiplicative character is computable on the subgroup of $K_{n}(A)$ generated by Loday products of elements in the connected component of the identity, $\mathrm{GL}_{0}(A)$.

Proof. Since each element in $\mathrm{GL}_{0}(A)$ can be obtained as a product of exponentials, the result follows by noting that the Loday product is multilinear and that the multiplicative character is a homomorphism of abelian groups.

In the case where the summability, $n=2 m$, of the Fredholm module $(F, H)$ is even, it is possible to express the above result in terms of the higher Helton-Howe trace form. Let $\mathcal{E} \subseteq \mathscr{L}(H)$ be the smallest $\mathbb{C}$-algebra of operators such that

$$
\mathscr{L}^{m}(H) \subseteq \mathcal{E} \quad \text { and } \quad P a P \in \mathcal{E} \quad \text { for all } a \in A .
$$

Here $P=(F+1) / 2 \in \mathscr{L}(H)$ is the projection onto the eigenvectors with eigenvalue 1 of the selfadjoint unitary $F \in \mathscr{L}(H)$. Since $A$ is assumed to be commutative all commutators between elements in $\mathcal{E}$ lie in the $m$-th Schatten ideal,

$$
[x, y] \in \mathscr{L}^{m}(H) \quad x, y \in \mathcal{E} .
$$

The higher Helton-Howe trace form on $\&$ is then given by

$$
\langle\cdot, \ldots, \cdot\rangle: \varepsilon^{2 m} \rightarrow \mathbb{C}, \quad\left\langle x_{1}, \ldots, x_{2 m}\right\rangle=\operatorname{Tr}\left(\sum_{s \in \Sigma_{2 m}} \operatorname{sgn}(s) x_{s(1)} \ldots x_{s(2 m)}\right) ;
$$

see [13], part II §2. In the paper [7], A. Connes proves the following equality, which relates the higher trace form to his index cocycle:

$$
\sum_{\mu \in \Sigma_{2 m-1}} \operatorname{sgn}(\mu) \tau_{F}\left(a_{0} \otimes a_{\mu(1)} \otimes \cdots \otimes a_{\mu(2 m-1)}\right)=-\frac{1}{m !}\left\langle P a_{0} P, \ldots, P a_{2 m-1} P\right\rangle .
$$

Here $a_{0}, \ldots, a_{2 m-1} \in A$ are any $2 m$ elements in $A$. Combining this identity with the above calculation we get a formula for the odd multiplicative character of Loday products involving the fundamental Helton-Howe trace form.

Corollary 5.4. Let $a_{0}, \ldots, a_{2 m-1} \in M_{\infty}(A)$. The odd multiplicative character of the Loday product $\left[e^{a_{0}}\right] * \cdots *\left[e^{a_{2 m-1}}\right] \in K_{2 m}(A)$ is then given by

$$
\begin{aligned}
\mathcal{M}_{F}\left(\left[e^{a_{0}}\right] * \cdots *\left[e^{a_{2 m-1}}\right]\right)= & -q\left(\frac{1}{m !}\left\langle P \operatorname{TR}\left(b_{0}\right) P, \ldots, P \operatorname{TR}\left(b_{2 m-1}\right) P\right\rangle\right) \\
& \in \mathbb{C} /(2 \pi i)^{m} \mathbb{Z} .
\end{aligned}
$$

Here $q: \mathbb{C} \rightarrow \mathbb{C} /(2 \pi i)^{m} \mathbb{Z}$ is the quotient map and $b_{0}, \ldots, b_{2 m-1} \in M_{\infty}(A)$ are any elements with

$$
e^{b_{i}}=e^{a_{i}} \quad \text { for all } i \in\{0, \ldots, 2 m-1\} .
$$




\section{References}

[1] A. J. Berrick, Characterisation of plus-constructive fibrations. Adv. in Math. 48 (1983), 172-176. Zbl 0531.55012 MR 700983

[2] L. G. Brown, The determinant invariant for operators with trace class self commutators. In Proceedings of a conference on operator theory (Proc. Conf., Dalhousie Univ., Halifax, N.S., 1973), Lecture Notes in Math. 345, Springer-Verlag, Berlin 1973, 210-228. Zbl 0284.47016 MR 0390830

[3] L. G. Brown, Operator algebras and algebraic K-theory. Bull. Amer. Math. Soc. 81 (1975), 1119-1121. Zbl 0332.46038 MR 0383090

[4] R. W. Carey and J. D. Pincus, An invariant for certain operator algebras. Proc. Nat. Acad. Sci. U.S.A. 71 (1974), 1952-1956. Zbl 0282.46056 MR 0344925

[5] R. W. Carey and J. D. Pincus, Almost commuting algebras. In $K$-theory and operator algebras (Proc. Conf., Univ. Georgia, Athens, Ga., 1975), Lecture Notes in Math. 575, Springer-Verlag, Berlin 1977, 19-43. Zbl 0358.46031 MR 0512491

[6] R. W. Carey and J. D. Pincus, Steinberg symbols modulo the trace class, holonomy, and limit theorems for Toeplitz determinants. Trans. Amer. Math. Soc. 358 (2006), 509-551. Zbl 1138.47302 MR 2177029

[7] A. Connes, Non-commutative differential geometry. Inst. Hautes Études Sci. Publ. Math. 62 (1985), 41-144. Zbl 0592.46056 MR 0823176

[8] A. Connes, Noncommutative geometry. Academic Press, San Diego, CA, 1994. Zbl 0818.46076 MR 1303779

[9] A. Connes and M. Karoubi, Caractère multiplicatif d'un module de Fredholm. K-Theory 2 (1988), 431-463. Zbl 0671.46034 MR 972606

[10] G. Cortiñas and A. Thom, Comparison between algebraic and topological $K$-theory of locally convex algebras. Adv. Math. 218 (2008), 266-307. Zbl 1142.19002 MR 2409415

[11] G. Ginot, Formules explicites pour le caractère de Chern en $K$-théorie algébrique. Ann. Inst. Fourier (Grenoble) 54 (2004), 2327-2355 (2005). Zbl 1068.19005 MR 2139695

[12] A. Grothendieck, Produits tensoriels topologiques et espaces nucléaires. Mem. Amer. Math. Soc. 16 (1955). Zbl 0064.35501 MR 0075539

[13] J. W. Helton and R. E. Howe, Integral operators: traces, index, and homology. In Proceedings of a conference on operator theory (Proc. Conf., Dalhousie Univ., Halifax, N.S., 1973), Lecture Notes in Math. 345, Springer-Verlag, Berlin 1973, 141-209. Zbl 0268.47054 MR 0390829

[14] C. E. Hood and J. D. S. Jones, Some algebraic properties of cyclic homology groups. $K$-Theory 1 (1987), 361-384. Zbl 0636.18005 MR 920950

[15] J. Kaad, Comparison of secondary invariants of finitely summable Fredholm modules. Submitted for publication.

[16] J. Kaminker, Algebraic $K$-theory invariants for operator theory. In Multivariable operator theory (Seattle, WA, 1993), Contemp. Math. 185, Amer. Math. Soc., Providence, RI, 1995, 187-194. Zbl 0831.46080 MR 1332060

[17] M. Karoubi, Homologie cyclique et $K$-théorie. Astérisque 149 (1987). Zbl 0648.18008 MR 913964 
[18] M. Karoubi, Sur la $K$-théorie multiplicative. In Cyclic cohomology and noncommutative geometry (Waterloo, ON, 1995), Fields Inst. Commun. 17, Amer. Math. Soc., Providence, RI 1997, 59-77. Zbl 0889.19001 MR 1478702

[19] M. Karoubi, Correspondance de Dold-Kan et formes differentielles. J. Algebra 198 (1997), 618-626. Zbl 0955.55011 MR 1489915

[20] J.-L. Loday, $K$-théorie algébrique et représentations de groupes. Ann. Sci. École Norm. Sup. (4) 9 (1976), 309-377. Zbl 0362.18014 MR 0447373

[21] J.-L. Loday, Cyclic homology. 2nd ed., Grundlehren Math. Wiss. 301, Springer-Verlag, Berlin 1998. Zbl 0885.18007 MR 1600246

[22] J.-L. Loday and D. Quillen, Cyclic homology and the Lie algebra homology of matrices. Comment. Math. Helv. 59 (1984), 569-591. Zbl 0565.17006 MR 780077

[23] J. P. May, Simplicial objects in algebraic topology. Chicago Lectures in Math., University of Chicago Press, Chicago 1992. Zbl 0769.55001 MR 1206474

[24] R. McCarthy, The cyclic homology of an exact category. J. Pure Appl. Algebra 93 (1994), 251-296. Zbl 0807.19002 MR 1275967

[25] D. Quillen, Higher algebraic $K$-theory: I. In Algebraic $K$-theory I-Higher $K$-theories (Proc. Conf., Battelle Memorial Inst., Seattle, Wash., 1972), Lecture Notes in Math. 341, Springer-Verlag, Berlin 1973, 85-147. Zbl 0292.18004 MR 0338129

[26] J. Rosenberg, Algebraic K-theory and its applications. Graduate Texts in Math. 147, Springer-Verlag, New York 1994. Zbl 0801.19001 MR 1282290

[27] J. Rosenberg, Comparison between algebraic and topological $K$-theory for Banach algebras and $C^{*}$-algebras. In Handbook of $K$-theory, Vol. 2, Springer, Berlin 2005, 843-874. Zbl 1123.19005 MR 2181834

[28] J. Stasheff, H-spaces from a homotopy point of view. Lecture Notes in Math. 161, Springer-Verlag, Berlin 1970. Zbl 0205.27701 MR 0270372

[29] U. Tillmann, Factorization of the (relative) Chern character through Lie algebra homology. K-Theory 6 (1992), 457-463. Zbl 0784.46057 MR 1194844

[30] B. L. Tsygan, Homology of matrix Lie algebras over rings and the Hochschild homology. Uspekhi Mat. Nauk 38 (1983), No. 2, 217-218; English transl. Russian Math. Surveys 38 (1983), No. 2, 198-199. Zbl 0526.17006 MR 695483

[31] B. Tsygan, Cyclic homology. Unpublished.

[32] J. B. Wagoner, Delooping classifying spaces in algebraic $K$-theory. Topology 11 (1972), 349-370. Zbl 0276.18012 MR 0354816

[33] C. A. Weibel, Nil $K$-theory maps to cyclic homology. Trans. Amer. Math. Soc. 303 (1987), 541-558. Zbl 0627.18005 MR 902784

Received April 23, 2009; revised December 7, 2009

J. Kaad, Mathematisches Institut, Universität Bonn, Endenicher Allee 60, 53115 Bonn, Germany

E-mail: jenskaad@hotmail.com 